\title{
A new enrichment space for the treatment of discontinuous pressures in multi-fluid flows
}

\author{
Roberto F. Ausas ${ }^{1}$, Gustavo C. Buscaglia ${ }^{1}$ and Sergio R. Idelsohn ${ }^{2, *, \dagger}$ \\ ${ }^{1}$ Instituto de Ciências Matemáticas e de Computação, Universidade de São Paulo, Av. do Trabalhador são-carlense \\ 400, Centro, 13566-590, São Carlos, SP, Brazil \\ ${ }^{2}$ ICREA Research Professor, Centro Internacional de Métodos Numéricos en Ingeniería., Edificio C1, Campus Norte \\ UPC C/ Gran Capitán S/N 08034 Barcelona, Spain
}

\begin{abstract}
SUMMARY
In this work, a new enrichment space to accommodate jumps in the pressure field at immersed interfaces in finite element formulations, is proposed. The new enrichment adds two degrees of freedom per element that can be eliminated by means of static condensation. The new space is tested and compared with the classical $P_{1}$ space and to the space proposed by Ausas et al (Comp. Meth. Appl. Mech. Eng., Vol. 199, 1019-1031, 2010) in several problems involving jumps in the viscosity and/or the presence of singular forces at interfaces not conforming with the element edges. The combination of this enrichment space with another enrichment that accommodates discontinuities in the pressure gradient has also been explored, exhibiting excellent results in problems involving jumps in the density or the volume forces. Copyright (C) 2011 John Wiley \& Sons, Ltd.
\end{abstract}

Received 16 May 2011; Revised 22 September 2011; Accepted 2 October 2011

KEY WORDS: multi-fluids; two-phase flows; embedded interfaces; finite element method; surface tension; discontinuous pressures; kinks

\section{INTRODUCTION}

The simultaneous presence of multiple fluids with varying properties in external or internal flows is found in daily life, marine environmental problems, and numerous industrial processes, among many other practical situations. Examples of these flows are gas-liquid transport, magma chambers, fluid-fuel interactions, crude oil recovery, spray cans, sediment transport in rivers and floods, pollutant transport in the atmosphere, cloud formation, fuel injection in engines, bubble column reactors and spray dryers for food processing, among others. This shows the importance of multifluid flows, which probably occur even more frequently than single phase flows [1]. As a result of the interaction between the different fluid components, multi-fluid flows are rather complex and very difficult to describe theoretically. For homogeneous flows, computational of fluid dynamics (CFD) has already a long history, and it is standard practice to use commercially available CFD codes. However, because of the complex physics involved in multi-fluid flows, the application of CFD in this area is rather young. Physical modeling in ad hoc laboratory scale models is not suitable for this purpose because of its complexity, the difficulty for scaling up to real life problems, and hence unaffordable costs. The alternative is, therefore, numerical modeling.

Despite the practical importance of the problem and the intensive work carried out in the last decade for the development of suitable mathematical and computational models, it is widely

\footnotetext{
*Correspondence to: Sergio R. Idelsohn, Centro Internacional de Métodos Numéricos en Ingeniería., Edificio C1, Campus Norte UPC C/ Gran Capitán S/N 08034 Barcelona, Spain.

${ }^{\dagger}$ E-mail: sergio@cimne.upc.edu 
accepted that the numerical study of heterogeneous flows is still a major challenge [1]. The complicated mathematical structure of the multi-fluid problem, multi-scale features of the flow, the existence of one or multiple internal interfaces and the unsteadiness of the flow, constitute major challenges for the analysis.

Computing the interface between various immiscible fluids or the free surfaces is difficult because neither the shape nor the positions of the interfaces are known a priori. The two approaches to solve these problems are: interface-tracking and interface-capturing methods. The former computes the motion of the flow particles via a Lagrangian approach where the computational domain adapts itself to the shape and position of the interfaces (see e.g., [2-7]). A different approach for the simulation of free-surface flows that is based on Lagrangian particles can be found in [8-11]. On the other hand, in the front-tracking method (see [12-14]), the interface is represented by a surface mesh advected with a Lagrangian method while immersed in an Eulerian (fix) mesh where the flow problem is solved considering the fluids as a single effective fluid with variable properties.

The other alternative is the interface-capturing method. Popular methods of this type are the volume-of-fluid technique (see [15-171) and the level set method (see, for example, [18-21]). In this case, the flow problem is also solved in a fixed underlying mesh considering a single fluid with variable properties. Variants of these methods mainly differ in two aspects: First, the technique used to solve the transport equation for the scalar function where the interface is embedded, for which a great deal of work has been carried out to improve accuracy for purely Eulerian methods [22-27] and for semi-Lagrangian methods [28-30]. Second, the technique used to solve the Navier-Stokes equations for a one-phase flow with variable properties and in how the fluiddynamical variables are treated near the interface because these can exhibit discontinuities in their values and/or their gradients owing to the discontinuities in the physical properties and/or the
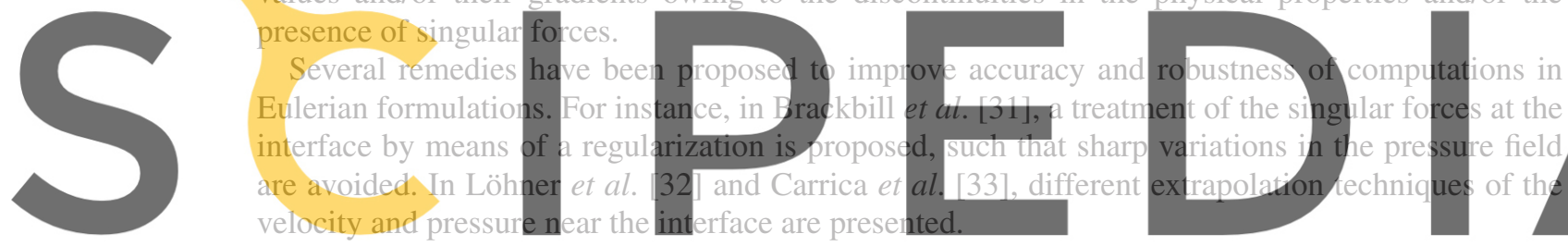

In this work, on the other hand, we focus the attention on how to improve the accuracy of simfrlations in finite, glement formulations by means of improving the approximation sipaces In this

not necessarily conform to the element edges, standard finite element methods, either continuous or

discontinuous across inter-element boundaries, suffer from suboptimal approximation orders. This poor approximation leads to spurious velocities near the interface that may significantly affect precision and robustness of numerical simulations (see e.g., [34]). One possibility is to locally modify the finite element spaces in those elements cut by the interface to accommodate the discontinuities. This can be carried out without introducing additional degrees of freedom, using the pressure space proposed by Ausas et al. [35]. The interpolation properties of this space are discussed in detail in [36], where the authors show that the interpolation order of this space is $\mathcal{O}\left(h^{\frac{3}{2}}\right)$ in the $L^{2}(\Omega)$-norm, which represents an improvement with respect to the order $\mathcal{O}\left(h^{\frac{1}{2}}\right)$ of the classical $P_{1}$-conforming space.

The other possibility that is explored in this article is to add degrees of freedom or enrich the finite element space at the elements cut by the interface. Minev et al. [37], and later Chessa and Belytschko [38], adopted an enrichment technique nowadays called XFEM, a name coined in the context of crack mechanics [39]. Both approaches lead to optimal orders of convergence, but the main drawback is that the additional degrees of freedom cannot be eliminated before assembly. The connectivity of the unknowns depend on the position of the interface, therefore, the mesh graph needs to be updated as the interface moves. Also, it has been observed that the resulting linear system becomes ill-conditioned because the linear independence of the finite element basis deteriorates as the mesh size is reduced. The XFEM approach has also been used recently in [40-42] for two-phase flows. A method that avoids the inclusion of additional degrees of freedom is the one presented by Fries et al. [43], which, on the other hand, has the drawback of the second-neighbor connectivity 
arising from the moving least square approach they use. Also, in [44], Codina and Coppola-Owen introduced an enrichment for the treatment of kinks in the pressure field as typically happens in problems with jumps in the density in the presence of a gravitational field. The additional degree of freedom can be statically condensed prior to assembly.

The main contribution of this paper is a new enrichment space for discontinuous pressures. Two enrichment functions are introduced at the elements crossed by the interface. The new functions are local to each element, linear on each side of the interface, discontinuous just at the interface and zero at the element nodes. The additional degrees of freedom can be condensed before assembly, avoiding the complexities associated with the update of the mesh graph. The implementation in any existing finite element code is extremely easy in two and three spatial dimensions, because the new shape functions are based on the usual $P_{1}$ functions. As illustrated in the numerical experiments, the interpolation properties of this new enrichment space are equal or better than those of the space presented in [35].

By way of outline, after this introduction, the governing equations and different jump conditions that arise in two-phase flows are recalled as well as the continuous and discrete variational formulations. Next, the new proposed enrichment space for discontinuous pressures is introduced with details to construct the enrichment functions. In Section 4, several problems in 2D and 3D are solved. Then, a further enrichment is proposed to deal with problems involving both discontinuities and kinks in the pressure field, in which, the new enrichment space is combined with the enrichment of [44]. Finally, some conclusions are drawn.
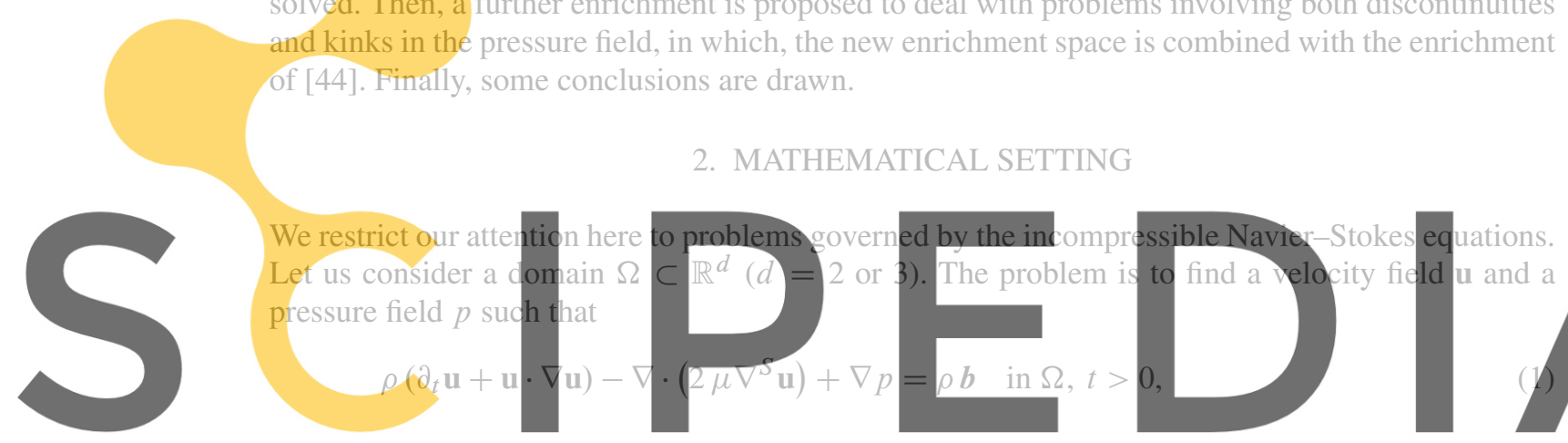

2. MATHEMATICAL SETTING

\section{Register for}
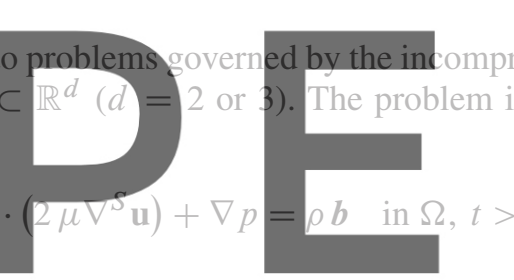

$$
\nabla \cdot \mathbf{u}=0 \text { in } \Omega, t>0 \text {, }
$$

free at https//www.scipedia.com

$$
\begin{gathered}
\mathbf{u}=\mathbf{u}_{\partial \Omega} \text { in } \partial \Omega, t>0, \\
\mathbf{u}=\mathbf{u}_{0} \quad \text { in } \Omega, t=0,
\end{gathered}
$$

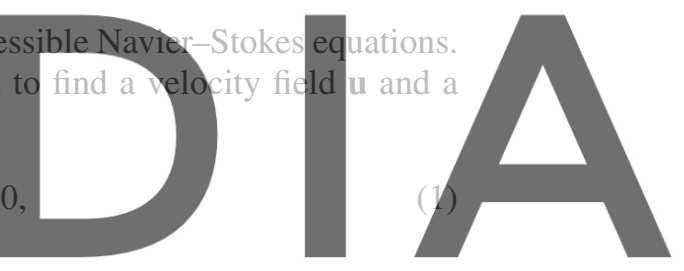

(2)

where $\mathbf{b}$ is a volume force, $\mathbf{u}_{\partial \Omega}$ are the Dirichlet boundary conditions and $\mathbf{u}_{0}$ is the initial condition for the velocity field. Although many phases can be present, we restrict here for the sake of simplicity to the case of two-phase flows. Denoting them by the plus ' + ' and minus ' - ' symbols, the fluid properties (density and viscosity) are given by

$$
(\rho(\mathbf{x}), \mu(\mathbf{x}))= \begin{cases}\left(\rho^{+}, \mu^{+}\right) & \text {if } \mathbf{x} \in \Omega^{+} \\ \left(\rho^{-}, \mu^{-}\right) & \text {if } \mathbf{x} \in \Omega^{-}\end{cases}
$$

The fluid domains $\Omega^{+}$and $\Omega^{-}$are separated by an interface denoted by $\Gamma$. In the following section, we comment on the boundary or jump conditions at this internal interface.

\subsection{Difficulties in the numerical solution of multi-phase flows-jump conditions}

In the numerical approximation of incompressible multi-fluid flows, several issues have to be considered:

1. a correct definition of the interface position;

2. possible pressure gradient jumps or kinks at the interface where density jumps are present;

3. possible pressure jumps at the interface where viscosity jumps are present; 
4. possible gradient velocity jumps where viscosity jumps are present; and

5. possible pressure jumps where surface tension is present.

Some of these aspects and possible numerical solutions have been previously discussed in [9] for Lagrangian formulations in which moving meshes are used. In this paper, we restrict our attention to items $2-5$ of the preceding list for the case of Eulerian formulations in which fixed meshes are used instead. Let us now see how these jumps appear in the different cases mentioned.

\section{Pressure gradient jumps}

Density jumps introduce a kink in the pressure field at the interface. This can be easily seen in the example of two fluids with different densities at rest, one on top of the other inside a closed cavity. The hydrostatic pressure gradient is discontinuous at the interface. Because in this case, the solution corresponds to an identically zero velocity field, the pressure gradient is simply given by

$$
\nabla p=\rho \mathbf{b},
$$

if the density has a jump at the interface, and the volume force is continuous, we have

$$
\llbracket \nabla p \rrbracket=\llbracket \rho \mathbf{b} \rrbracket=\llbracket \rho \rrbracket \mathbf{b}=\left(\rho^{+}-\rho^{-}\right) \mathbf{b},
$$

where $\llbracket \cdot \rrbracket$ represents the jump of any quantity at the interface. A similar situation can occur in the case of a discontinuous volume force. This could be the case of an electric force acting just on one of the fluids, having for instance distributed electrical charges. As mentioned earlier, a remedy for this problem is the enrichment proposed in [44], which is later recalled.
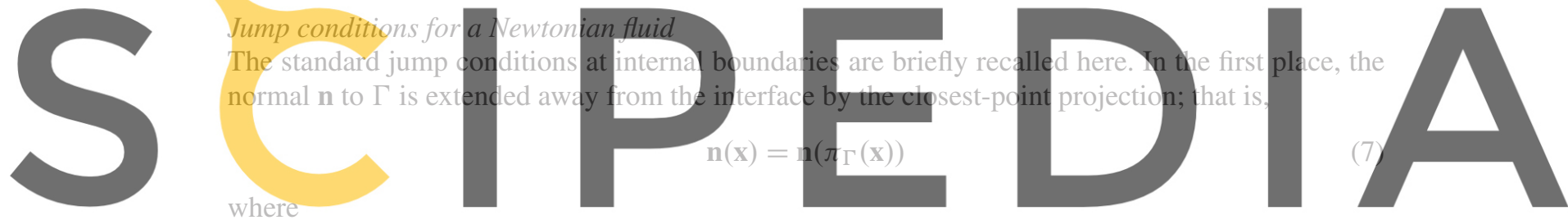

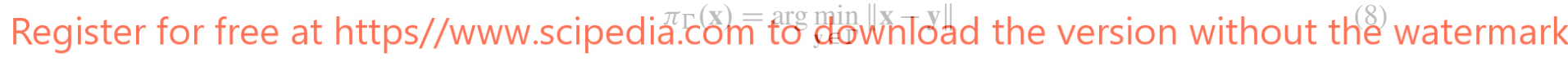

This allows to decompose the velocity field u near the interface into its normal and tangential parts,

$$
\mathbf{u}=u_{n} \mathbf{n}+\mathbf{u}_{s},
$$

where $u_{n}=\mathbf{u} \cdot \mathbf{n}$. The interface forces must balance the sum of the forces exerted on $\Gamma$ from the 'positive' side, plus those from the 'negative' side. This is expressed as

$$
\left(\sigma^{-}-\sigma^{+}\right) \cdot \mathbf{n}=\mathbf{f}_{\Gamma} .
$$

Now, for the normal component of this jump, we have

$$
\left[\left(\sigma^{-}-\sigma^{+}\right) \cdot \mathbf{n}\right] \cdot \mathbf{n}=\llbracket \sigma_{n n} \rrbracket=\llbracket-p+2 \mu \frac{\partial u_{n}}{\partial n} \rrbracket=f_{\Gamma_{n}} .
$$

For the tangential component, we project the right hand side of Equation (10) onto the tangent plane to the interface

$$
\begin{aligned}
\Pi_{\Gamma}\left[\left(\sigma^{-}-\sigma^{+}\right) \cdot \mathbf{n}\right] & =\llbracket \mu\left(\frac{\partial \mathbf{u}_{s}}{\partial n}+\nabla_{\Gamma} u_{n}-\mathbb{H} \cdot \mathbf{u}_{s}\right) \rrbracket= \\
& =\llbracket \mu \frac{\partial \mathbf{u}_{s}}{\partial n} \rrbracket+\llbracket \mu \rrbracket\left(\nabla_{\Gamma} u_{n}-\mathbb{H} \cdot \mathbf{u}_{s}\right)=\mathbf{f}_{\Gamma s},
\end{aligned}
$$

where $\Pi_{\Gamma}$ is the tangential projector operator, that for a given vector $\mathbf{w}$ is given by

$$
\Pi_{\Gamma} \mathbf{w}=\mathbf{w}_{s}=\mathbf{w}-w_{n} \mathbf{n}=(\mathbb{I}-\mathbf{n} \otimes \mathbf{n}) \mathbf{w},
$$


The symbol $\nabla_{\Gamma}$ is the surface gradient, which, for a function $f$ defined on $\Gamma$, is given by

$$
\nabla_{\Gamma} f=\Pi_{\Gamma} \nabla \tilde{f}
$$

where $\tilde{f} \in \mathcal{C}^{1}$ is any extension out of $\Gamma$ of $f$. Finally, $\mathbb{H}\left(=\nabla_{s} \mathbf{n}\right)$ is a symmetric tensor called the second fundamental form. This tensor contains the geometrical information of the interface. In particular, its trace is the well known mean curvature $\kappa$. For a quick review of these formulae from differential geometry in the context of capillary flows, the reader is referred to the recent article by Buscaglia and Ausas [45].

Remark: In the general case, both the pressure and the velocity gradient can be discontinuous at the interface. However, for problems involving surface tension, in the absence of Marangoni or thermocapillary effects, the force $\mathbf{f}_{\Gamma}$ is normal to the interface and given by

$$
\mathbf{f}_{\Gamma}=\gamma \kappa \mathbf{n},
$$

where $\gamma$ is the (constant) surface tension coefficient. If viscosities of both fluids are the same, just the pressure exhibits a jump. In the numerical solution of problem (1)-(4) with jump conditions (6), (11) and (12), regardless of the method used (finite elements, finite differences, etc.), special care has to be taken to accommodate the discontinuities in the fluid-dynamical variables near the interface. In this article, we use a finite element method of which the variational formulation is presented succeedingly. In this case, the key issue relies on how to choose the approximation spaces for pressure and velocity.

\subsection{Variational formulation}
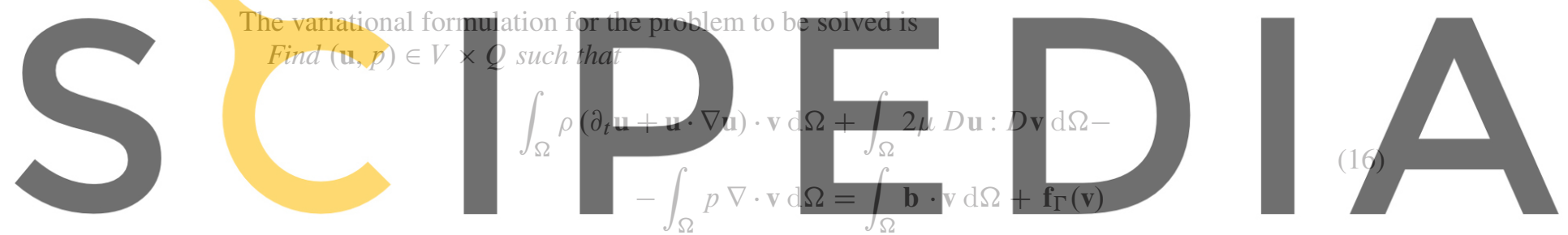

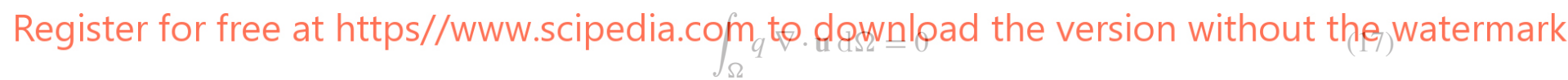

$\forall(v, q) \in V \times Q$. The term $f_{\Gamma}(v)$ in Equation (16) represents the contribution of a singular force concentrated at the interface $\Gamma$. For the case of surface tension, we use a Laplace-Beltrami formulation (see e.g., [34,40,45,46]), that is,

$$
\mathbf{f}_{\Gamma}(\mathbf{v})=-\int_{\Gamma} \gamma(\mathbf{x})(\mathbb{I}-\mathbf{n} \otimes \mathbf{n}): \nabla \mathbf{v} d \Gamma
$$

that accounts for both,surface tension and Marangoni effects.

We use a stabilized formulation based on the ASGS (Algebraic Subgrid Scale) method for discretization (see, e.g., [47] and references therein) together with a trapezoidal rule for temporal discretization and a monolithic approach solving simultaneously for velocity and pressure using a Newton-Raphson iterative method. The discrete variational formulation of this problem then reads

Find $\left(\mathbf{u}_{h}^{n+1}, p_{h}^{n+1}\right) \in V_{h} \times Q_{h}$ such that

$$
\begin{gathered}
\mathcal{R}_{\mathbf{u}}=\int_{\Omega} \mathcal{G}_{\mathbf{u}} \cdot \mathbf{v}_{h} \mathrm{~d} \Omega+\int_{\Omega} 2 \mu \nabla^{S} \mathbf{u}_{h}^{n+1}: \nabla \mathbf{v}_{h} \mathrm{~d} \Omega-\int_{\Omega} p_{h}^{n+1} \nabla \cdot \mathbf{v}_{h} \mathrm{~d} \Omega+\mathbf{f}_{\Gamma_{h}}^{n+1}\left(\mathbf{v}_{h}\right)+ \\
+\sum_{K \in \mathcal{T}_{h}} \tau_{K} \int_{\Omega_{K}}\left(\mathcal{G}_{\mathbf{u}}+\nabla p_{h}^{n+1}\right) \cdot \mathbf{u}_{h}^{n} \cdot \nabla \mathbf{v}_{h}+\sum_{K \in \mathcal{T}_{h}} \int_{\Omega_{K}} \delta_{K} \nabla \cdot \mathbf{u}_{h}^{n+1} \nabla \cdot \mathbf{v}_{h} \mathrm{~d} \Omega=0 \\
\mathcal{R}_{p}=\int_{\Omega} q_{h} \nabla \cdot \mathbf{u}_{h}^{n+1} \mathrm{~d} \Omega+\sum_{K \in \mathcal{T}_{h}} \int_{\Omega_{K}} \frac{\tau_{K}}{\rho}\left(\mathcal{G}_{\mathbf{u}}+\nabla p_{h}^{n+1}\right) \cdot \nabla q_{h} \mathrm{~d} \Omega=0
\end{gathered}
$$


$\forall\left(\mathbf{v}_{h}, q_{h}\right) \in V_{h} \times Q_{h}$. In (19), the term $\mathcal{G}_{\boldsymbol{u}}$ is given by

$$
\mathcal{G}_{\mathbf{u}}=\rho\left(\frac{\mathbf{u}_{h}^{n+1}-\mathbf{u}_{h}^{n}}{\Delta t}+\mathbf{u}_{h}^{n+1} \cdot \nabla \mathbf{u}_{h}^{n+1}-\boldsymbol{b}^{n+1}\right),
$$

with $\Delta t$ the time step and the stabilization parameters given by

$$
\tau_{K}=c\left[4 \frac{v}{h^{2}}+2 \frac{\left|\mathbf{u}_{h}\right|_{\infty}^{n}}{h}\right]^{-1}, \quad \delta_{K}=2 \mu+\rho\left|\mathbf{u}_{h}\right|_{\infty}^{n} h_{K}
$$

where $c$ is a tuning parameter, that for the academic problems shown in Section 4 is taken equal to 1 , whereas for the $3 \mathrm{D}$ examples, is taken equal to 0.1 .

Many authors introduce a regularized form of the Dirac delta function for the computation of $\mathbf{f}_{\Gamma_{h}}^{n+1}\left(\mathbf{v}_{h}\right)$ in (19) as proposed in $[31,48]$, therefore, the surface force is approximated as a volume force more or less concentrated around $\Gamma$ depending on an adjustable regularization parameter. In our formulation, we do not use such regularizations and instead compute this term exactly, which precisely leads to sharp variations through the interface in the pressure field. Solving problem (19)-(20) accuratelly, requires the finite element spaces to be appropriately chosen, so as to accomodate discontinuities and/or kinks in the pressure and velocity fields. We first focus on how to improve the accuracy of the numerical approximation in several problems involving jumps in the pressure field, for which we introduce a new enrichment space.

\section{NEW ENRICHMENT SPACE FOR DISCONTINUOUS PRESSURES}

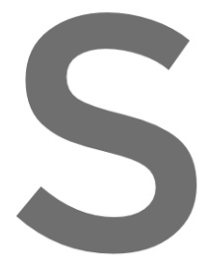

First, consider a finite and tetrahedra in the tices per element is $\Gamma_{h}$ is composed of element edges. The a typical element cut by $\Gamma_{h}$ in two and three spat
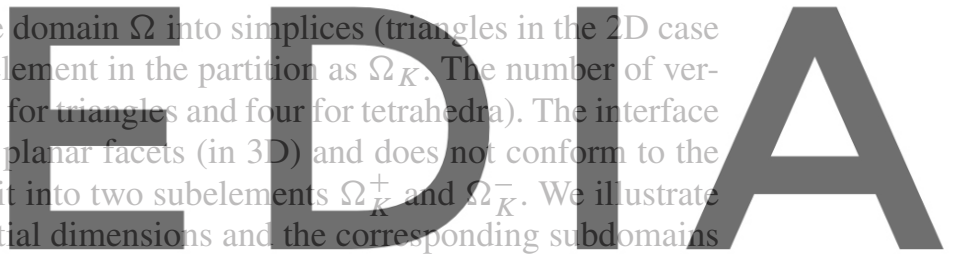

in Figure 1. Those cases in which the interface passes through the nodes are degenerate cases that

the discrete variational formulation presented earlier, the integrals over the elements are performed exactly just by redefining the quadrature rule in the elements cut by the interface after subdivision following $\Gamma_{h}$. The velocity field is made up of continuous linear functions. For all the elements of $\mathcal{T}_{h}$ that are not crossed by $\Gamma_{h}$, the space $Q_{h}$ is also made up of continuous linear functions. The various possibilities to improve accuracy of the numerical approximation in problems involving jumps or kinks, consist in an enrichment or a modification of the finite element space $Q_{h}$ only in those elements of $\mathcal{T}_{h}$ crossed by $\Gamma_{h}$.

The discrete pressure proposed here can be written as

$$
p_{h}=\sum_{J \in \mathcal{J}} P_{J} N_{J}+\sum_{J \in \mathcal{J}_{e}} C_{J} M_{J}
$$

where $\mathcal{J}=\left\{1, \ldots, n_{P}\right\}$, with $n_{P}$ the number of standard degrees of freedom per element and $\mathcal{J}_{e}$ the set of indices accounting for the additional enrichment functions local to the element.

To build the new pressure space, a requirement for the enrichment is that a constant solution on each fluid domain with a jump at the interface belong to the discrete space $Q_{h}$. To satisfy this requirement, we introduce two new enrichment functions that are linear on each subelement $\Omega_{K}^{+}$and $\Omega_{K}^{-}$and discontinuous at $\Gamma_{h}$. We also require both functions to be zero at the nodes of the simplex. By inspection, we see that a possibility to define the enrichment functions is as follows:

$$
\begin{gathered}
M_{1}(\mathbf{x})=(1-S(\mathbf{x})) \chi^{+}(\mathbf{x}), \\
M_{2}(\mathbf{x})=S(\mathbf{x}) \chi^{-}(\mathbf{x}),
\end{gathered}
$$




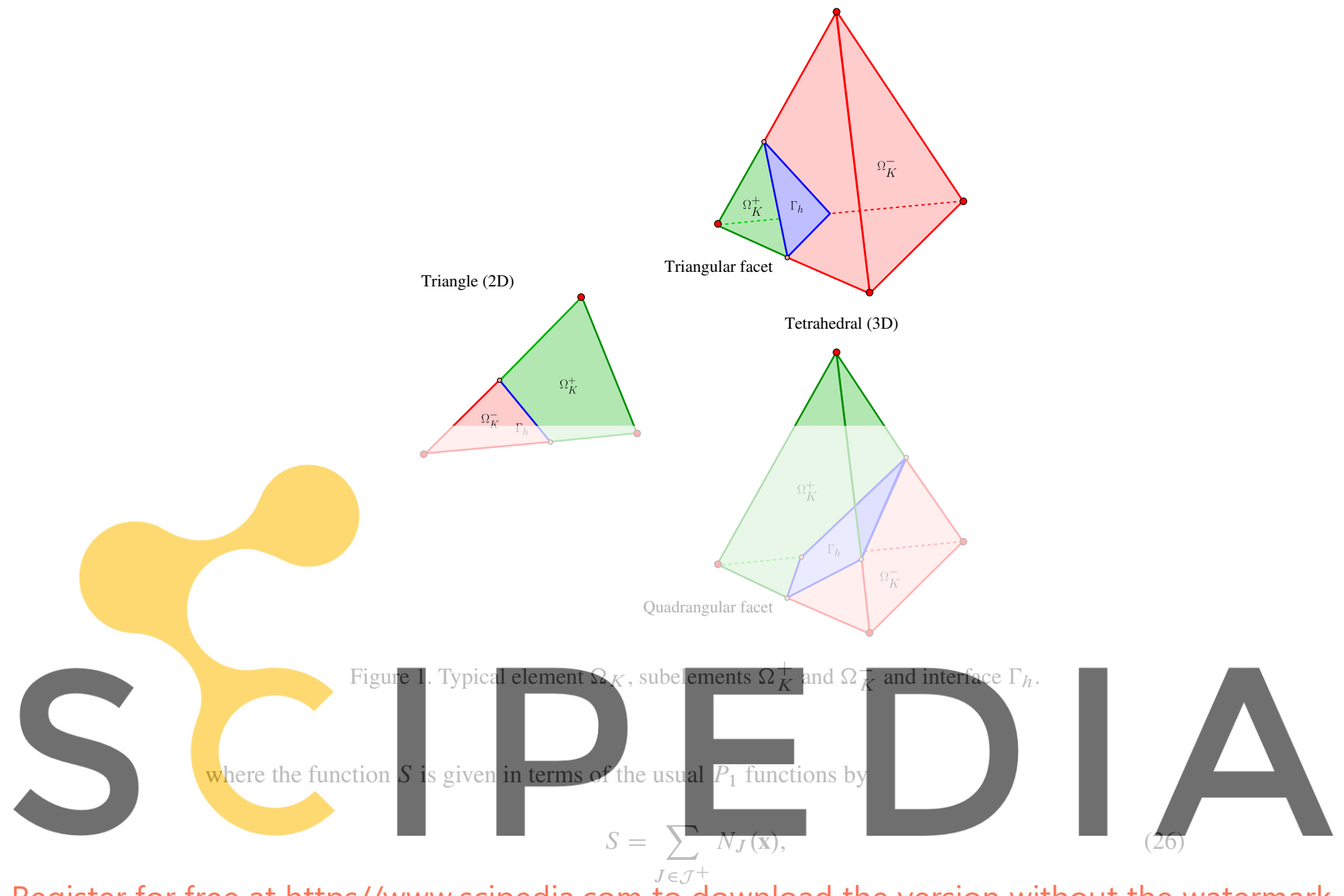

Register for free at https//www.scipedia.com to download the version without the watermark

with $\mathcal{J}^{+}=\left\{J \in \mathcal{J}, \mathbf{x}_{J} \in \Omega_{K}^{+}\right\}$, and $\chi^{+}$and $\chi^{-}$the characteristic functions for the positive and negative sides. The dimension of the pressure space is thus dim $Q_{h}=N_{P}+2 N_{E}$, where $N_{P}$ and $N_{E}$ are the total number of nodes and elements respectively in $\mathcal{T}_{h}$. However, because the additional shape functions are local to each element crossed by the interface, they can be condensed prior to assembly, and the size of the final linear system to be solved is the same as in the standard case. Note that this elimination can be carried out because the pressure is just involved in linear terms of the problem.

In Figure 2, the enrichment functions for a typical triangular element are shown. In the left part of the figure, the two functions are shown separately, and in the right part, they are plotted together just for illustrative purposes. In Table I, the computation of these functions is given in the form of a pseudo-code.

\subsection{Static elimination of the enrichment unknowns}

Before final assembly, the linear system to be solved at each non-linear iteration can be written by blocks as follows

$$
\left[\begin{array}{cc}
\mathrm{A}_{S S} & \mathrm{~A}_{S M} \\
\mathrm{~A}_{M S} & \mathrm{~A}_{M M}
\end{array}\right]\left[\begin{array}{l}
\delta \mathbf{X}_{S} \\
\mathbf{X}_{M}
\end{array}\right]=-\left[\begin{array}{c}
\mathcal{R}_{S} \\
\mathcal{F}_{M}
\end{array}\right]
$$

where the subindex $S$ refers to the velocity and standard pressure degrees of freedom and the subindex $M$ refers to the additional degrees of freedom. At the elementary level the dimension 


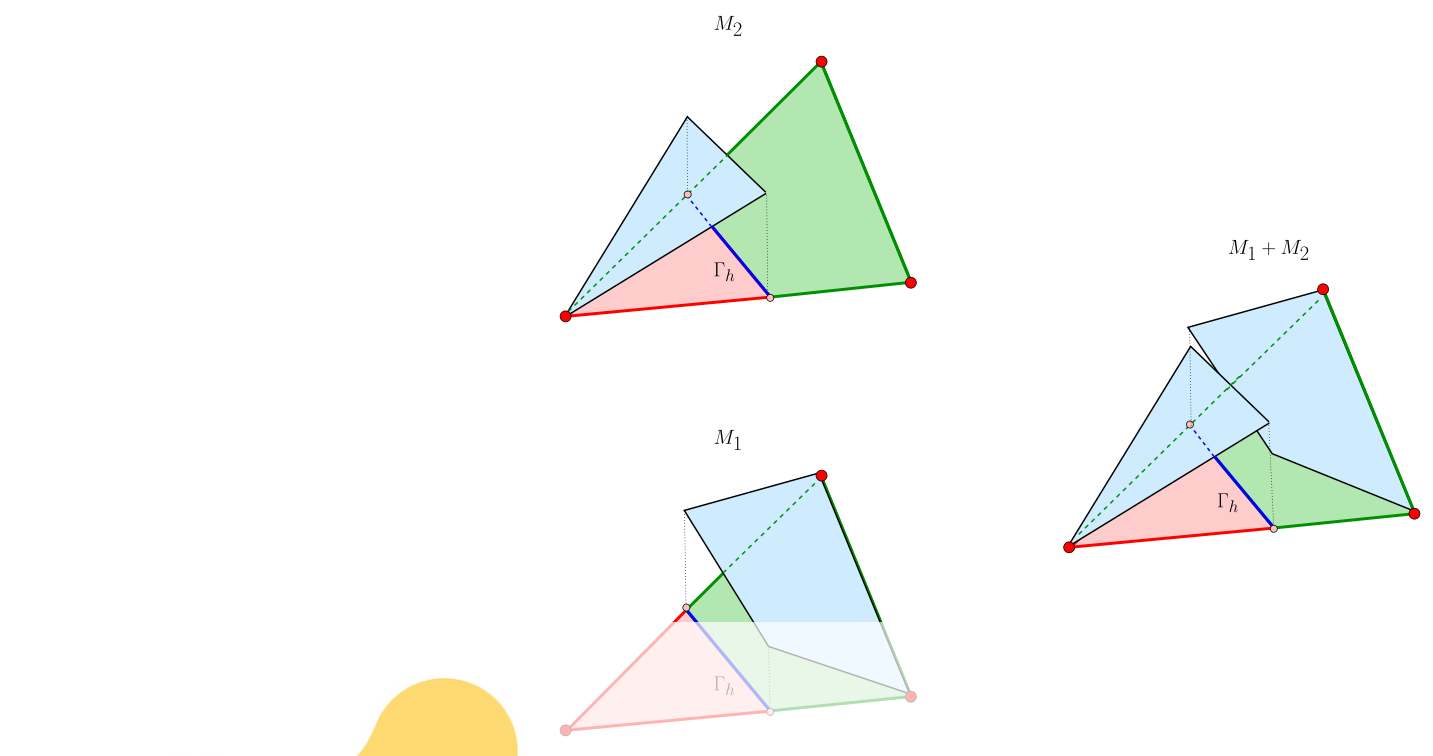

Figure 2. New enrichment functions in a typical triangular element. On the left, the two functions are drawn separately. On the right, the two functions are drawn together just for illustrative purposes.
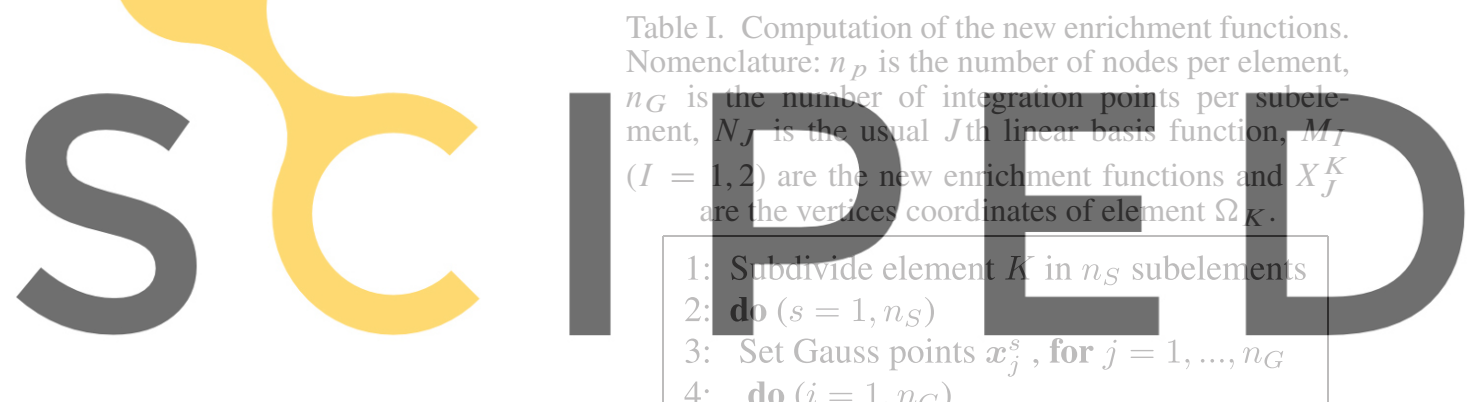

Table I. Computation of the new enrichment functions.

Nomenclature: $n_{p}$ is the number of nodes per element,
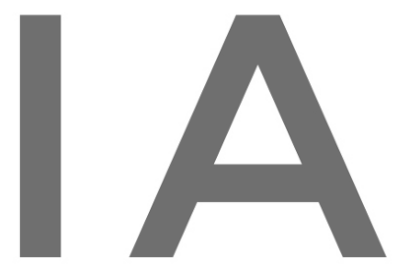

Register for free at https//www.ss!pegdia. $g_{2}, n_{G}$ to download the version without the watermark

$$
\begin{aligned}
& \text { 6: } \quad \text { do }\left(J=1, n_{p}\right) \\
& \text { 7: } \quad \text { if }\left(\boldsymbol{X}_{J}^{K} \in \Omega_{K}^{+}\right) \\
& \text {8: } \quad \text { Set } S\left(\boldsymbol{x}_{i}^{s}\right) \leftarrow S\left(\boldsymbol{x}_{i}^{s}\right)+N_{J}\left(\boldsymbol{x}_{i}^{s}\right) \\
& \text { 9: } \quad \text { end if } \\
& \text { 10: } \quad \text { end do } \\
& \text { 11: if }\left(\boldsymbol{x}_{i}^{s} \in \Omega_{K}^{+}\right) \\
& \text {12: } \quad \text { Set } M_{1}\left(\boldsymbol{x}_{i}^{s}\right)=1-S\left(\boldsymbol{x}_{i}^{s}\right) \\
& \text { 13: } \quad \text { Set } M_{2}\left(\boldsymbol{x}_{i}^{s}\right)=0 \\
& \text { 14: } \quad \text { else } \\
& \text { 15: } \quad \text { Set } M_{1}\left(\boldsymbol{x}_{i}^{s}\right)=0 \\
& \text { 16: } \quad \text { Set } M_{2}\left(\boldsymbol{x}_{i}^{s}\right)=S\left(\boldsymbol{x}_{i}^{s}\right) \\
& \text { 17: end if } \\
& \text { 18: } \quad \text { end do } \\
& \text { 19: end do }
\end{aligned}
$$

of matrix $\mathrm{A}_{S S}$ is $n_{p}^{2} \times n_{p}^{2}$, of matrix $\mathrm{A}_{S M}$ is $n_{p}^{2} \times 2$, of matrix $\mathrm{A}_{M S}$ is $2 \times n_{p}^{2}$ and of matrix $\mathrm{A}_{M M}$ is $2 \times 2$ and its $I J$ entry is given by

$$
\mathrm{A}_{M M}^{I J}=\frac{\tau_{K}}{\rho} \int_{\Omega_{K}} \nabla M_{I} \cdot \nabla M_{J} \mathrm{~d} \Omega
$$


which for our case is a positive definite diagonal matrix because note that $M_{1}=0$ and $M_{2} \neq 0$ in $\Omega_{K}^{-}$and $M_{1} \neq 0$ and $M_{2}=0$ in $\Omega_{K}^{+}$. For the additional degrees of freedom, we do not compute the incremental values, but the unknown $\mathbf{X}_{M}$ because the pressure is just involved in the linear terms of the problem. Now, using the fact that the enrichment functions are local to each element, we eliminate $\mathbf{X}_{M}$ at the elementary level before final assembly as follows

$$
\left[\mathrm{A}_{S S}-\mathrm{A}_{S M}\left(\mathrm{~A}_{M M}\right)^{-1} \mathrm{~A}_{M S}\right] \delta \mathbf{X}_{S}=-\mathcal{R}_{S}+\mathrm{A}_{S M}\left(\mathrm{~A}_{M M}\right)^{-1} \mathcal{F}_{M}
$$

The enrichment shape functions can be normalized to avoid round-off errors in the computation of $\left(\mathrm{A}_{M M}\right)^{-1}$. The normalization factor can be defined simply by doing an order of magnitude estimation. Because, each entry of $A_{M M}$ is the product of derivatives of two linear shape functions of order $\sim 1 / h^{2}$ that are integrated over the element volume, which is of order $\sim h^{d}$ and the stabilization $\tau_{K} / \rho \sim h^{2} / \mu$ (considering the viscous dominated case for simplicity), the normalization factor can be chosen to be of order $h^{d} / \mu$ so as to end up with entries of order 1 in matrix $\mathrm{A}_{M M}$.

Numerical results using the new enrichment space will be compared with the classical $P_{1}$ conforming space and with the space presented in [35], which is recalled here for completeness.

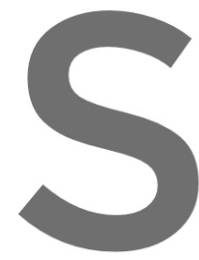

3.2. Space of [35] for discontinuous pressures without additional unknowns

The interpolation $\mathrm{p}$ space, no additional ified so as to capture computed element-bydiscontinuous at the one at their correspon The interpolation properties of the space proposed in [35] hive also been discussed in [36]. In this
space, no additional degrees of freedony are incorpdrated but the pressure shape functions are mod-
ified so as to capture discontinuities in the pressure field. The modifications are local and can be
computed element-by-element. The modified functions are piecewise linear on each side and only
discontinuous at the interface. $\Gamma$ h. They form a nodal basis, in the sense that they take the value
one at their corresponding node and zero at the other nodes. To define them, we simply 'cary'
the value at each node towards the intersection of any edge emanating from it with the interface.
The shape functions for a typical triangular element are shown in Figure 3 , but again, the func-

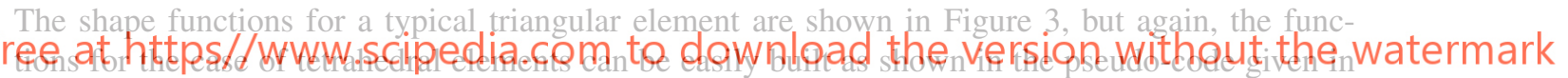
Register for freesato
Table II.
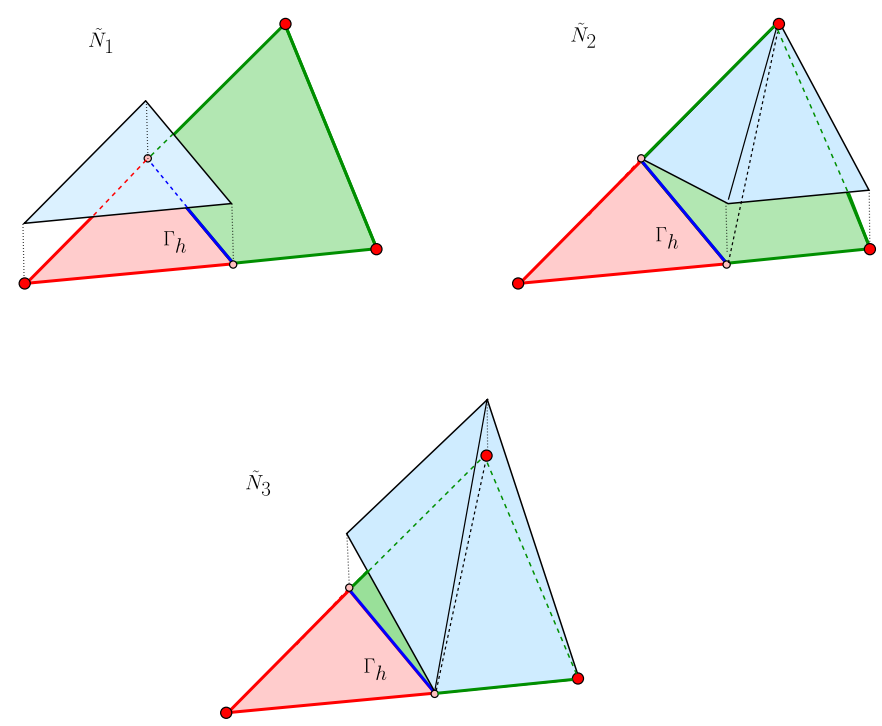

Figure 3. Finite element basis shape functions for the space proposed in [35] in a typical triangular element. 
Table II. Computation of the basis functions for the space of [35]. Nomenclature: $n_{p}$ is the number of nodes per element, $n_{G}$ is the number of integration points per subelement, $N_{J}$ is the usual $J$ th linear basis function, $\tilde{N}_{I}(I=1,2,3)$ are the modified basis functions and $\boldsymbol{X}_{I}^{K}$ are the vertices coordinates of element $\Omega_{K}$.

\begin{tabular}{|c|}
\hline 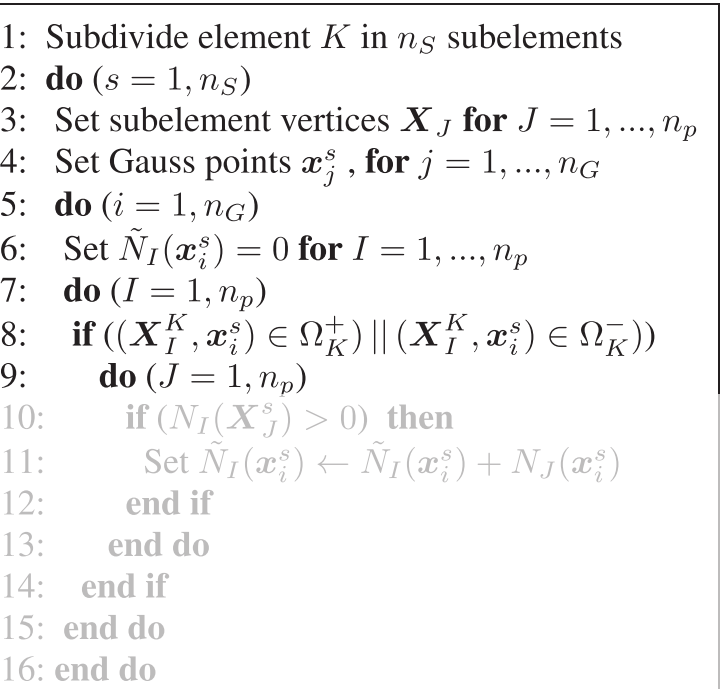 \\
\hline
\end{tabular}
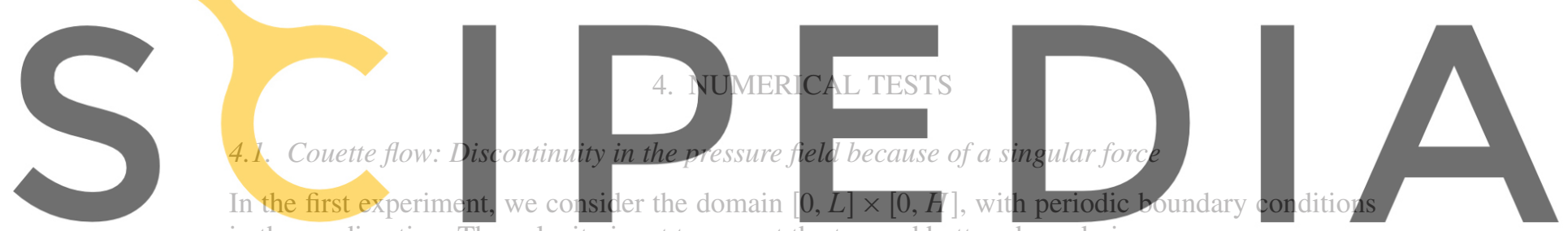

in the $x_{1}$-direction. The velocity is set to zero at the top and bottom boundaries

\section{Register for free at https//www.scipediax.cem toudowbloted the version without the watermark}

and the interface $\Gamma$ is the straight vertical line $x_{1}=a$, on which a constant unit normal force $f=1$ is imposed. The exact solution for this probiem is

$$
\begin{aligned}
& u_{1}\left(x_{1}, x_{2}\right)=\frac{1}{2 \mu L} x_{2}\left(H-x_{2}\right) \\
& u_{2}\left(x_{1}, x_{2}\right)=0 \\
& p\left(x_{1}, x_{2}\right)=-\frac{1}{L} x_{1}+\mathcal{H}\left(x_{1}-a\right)
\end{aligned}
$$

The indeterminacy of the pressure is removed by imposing $p(0,0)=0$ instead of setting the average to zero, for simplicity. This problem, with $L=3, H=1, \mu=1$ and $a=2$ was discretized with the stabilized equal-order formulation presented earlier with the classical $P_{1}$-conforming pressure space, the space of [35] and the new enrichment space.

A sequence of unstructured meshes was built, of which the first one is shown in Figure 4. To this mesh, which consists of 3520 triangles, we assign a mesh size of $h=0.044$. The following meshes in the sequence are built by subdivision. We measure the velocity error in the $H^{1}(\Omega)$-norm and the pressure error in the $L^{2}(\Omega)$-norm as functions of $h$ for all three pressure spaces. The results of the convergence analysis are displayed in Figures 5 and 6 . As seen in the figures, the error is very similar for both discontinuous spaces. The pressure field corresponding to the classical $P_{1^{-}}$conforming pressure is compared with that obtained using the new enrichment space in Figure 7. 


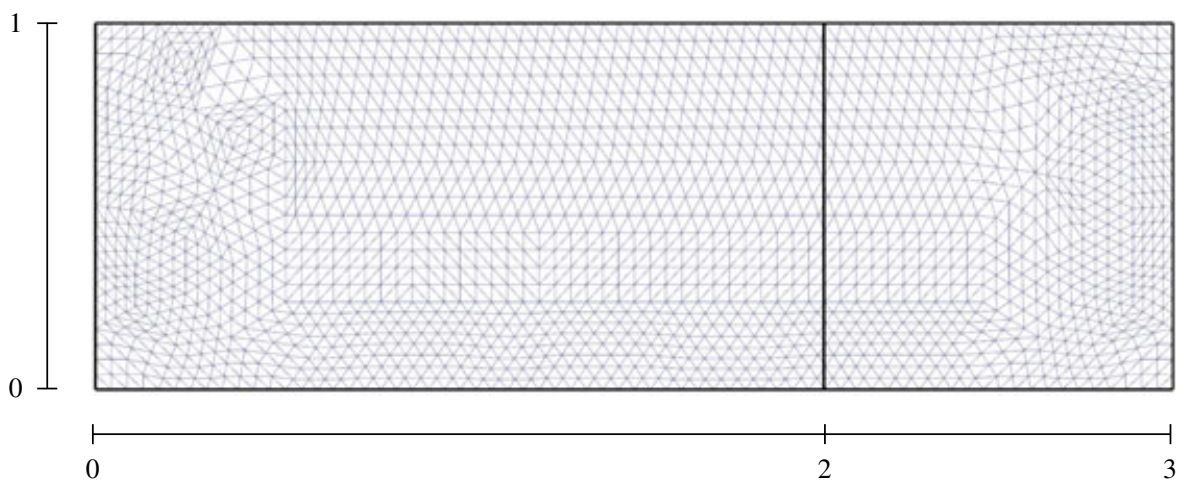

Figure 4. Mesh for the Couette flow problem convergence study, with 3520 elements and $h=0.044$.

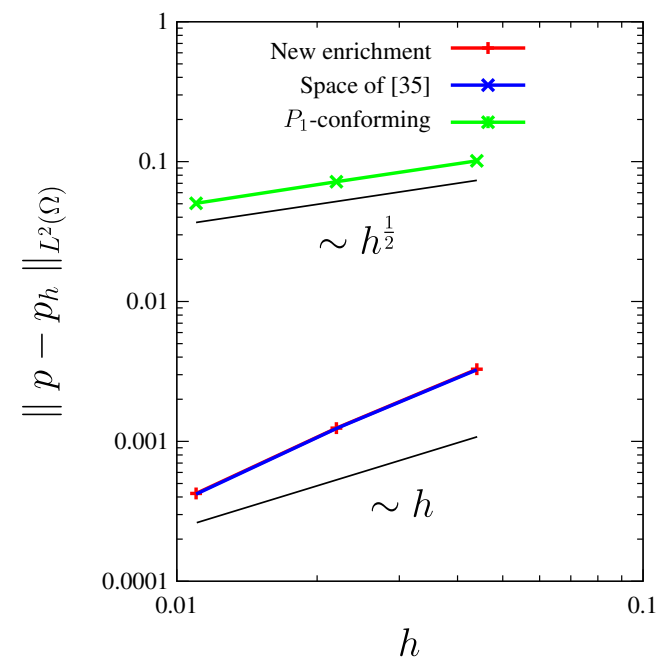

Figure 5. Error norm for the pressure field, showing the convergence rates for the Couette flow problem.

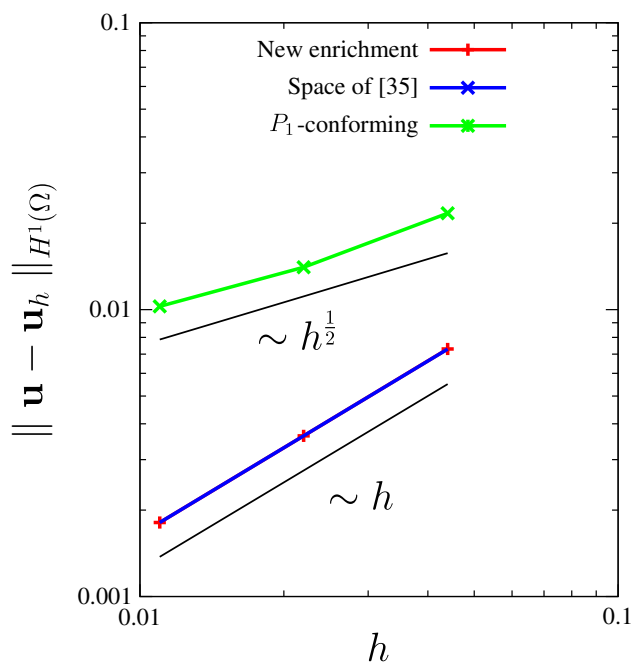

Figure 6. Error norm for the velocity field, showing the convergence rates for the Couette flow problem. 


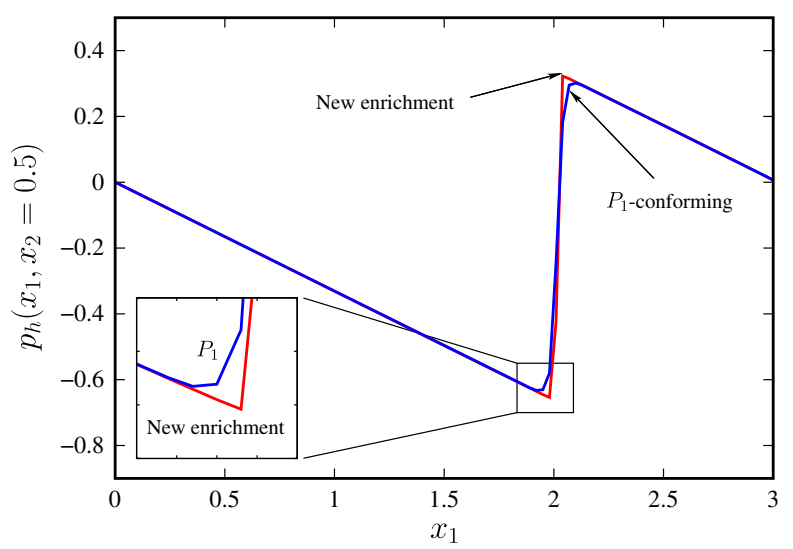

Figure 7. Pressure field section at $y=0.5$ for the Couette flow problem.

From the figure, we see that the new enrichment space exhibits significantly better behavior near the interface than the classical $P_{1}$-conforming space. Results obtained using the space of [35] are similar to those corresponding to the new space.

\subsection{Extensional flow: discontinuity in the pressure field because of a jump in the viscosity}

We consider an extensional flow problem in which the computational domain $[0,1] \times[0,1]$ is split by an interface separating fluids with different viscosities $\mu_{1}$ and $\mu_{2}$. The density $\rho$ is assumed the same for both fluids. In this case, the interface $\Gamma$ is the straight horizontal line $x_{2}=a$. Considering the following linear velocity field

$$
\begin{gathered}
u_{1}\left(x_{1}, x_{2}\right)=1-x_{1}, \\
u_{2}\left(x_{1}, x_{2}\right)=x_{2},
\end{gathered}
$$

and neglecting the volume forces, it can be easily found that the exact solution for the pressure field is quadratic on each fluid and with a jump at the interface because of the viscosity difference. This pressure field is given by

$$
p\left(x_{1}, x_{2}\right)=\rho\left(x_{1}-\frac{1}{2}\left(x_{1}^{2}+x_{2}^{2}\right)\right)+2\left(\mu_{1}-\mu_{2}\right) \mathcal{H}\left(a-x_{2}\right)
$$

where $\mathcal{H}\left(a-x_{2}\right)=1$ if $x_{2}<a$ and zero otherwise. The indeterminacy of the pressure in the simulations is again removed by imposing $p(1,1)=0$ instead of setting the average to zero. To reproduce this exact solution, the velocity field given by Equations (33)-(34) is imposed at the boundaries.

The problem is solved with $\rho=10, \mu_{1}=5$ and $\mu_{2}=1$ and $a=0.5$ using the classical $P_{1}$-conforming pressure space, the new enrichment space and the space of [35]. A sequence of unstructured meshes was built, of which the first one is shown in Figure 8. To this mesh, which consists of 832 triangles, we assign a mesh size of $h=0.055$. The following meshes in the sequence are built by subdivision of each triangle into four equal triangles. We measure the velocity error in the $H^{1}(\Omega)$-norm and the pressure error in the $L^{2}(\Omega)$-norm as function of $h$. The results of the convergence analysis are displayed in Figures 9 and 10. Results for the new enrichment space exhibit, in this case a much smaller error (more than one order of magnitude) than results for the space of [35], in both pressure and velocity. Also, for the meshes considered, we observe a better convergence order of the new enrichment space, equal to $h^{2}$ for pressure and $h^{\frac{5}{2}}$ for velocity.

In Figure 11, the pressure field for the the new enrichment space (left) and the classical $P_{1^{-}}$conforming pressure space (right) are shown. In Figure 12, cuts of these pressure fields at $x_{1}=0.5$ are compared, from which we clearly appreciate the better behavior near the interface when the new enrichment space is used. Note that the case without inertial effects (i.e., $\rho=0$ ) corresponds to a constant pressure field on each fluid, with a jump at the interface of magnitude $2\left(\mu_{1}-\mu_{2}\right)$. This 


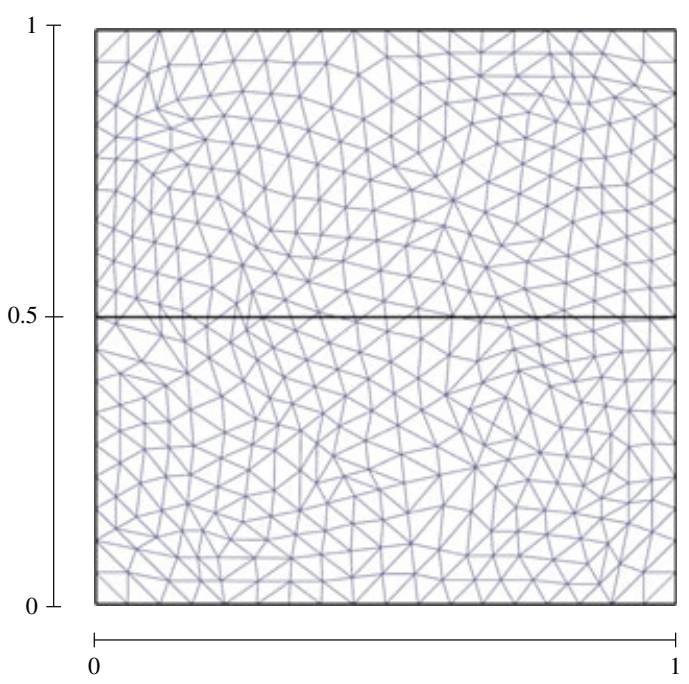

Figure 8. Mesh for the extensional flow problem convergence study, with 832 elements and $h=0.055$.

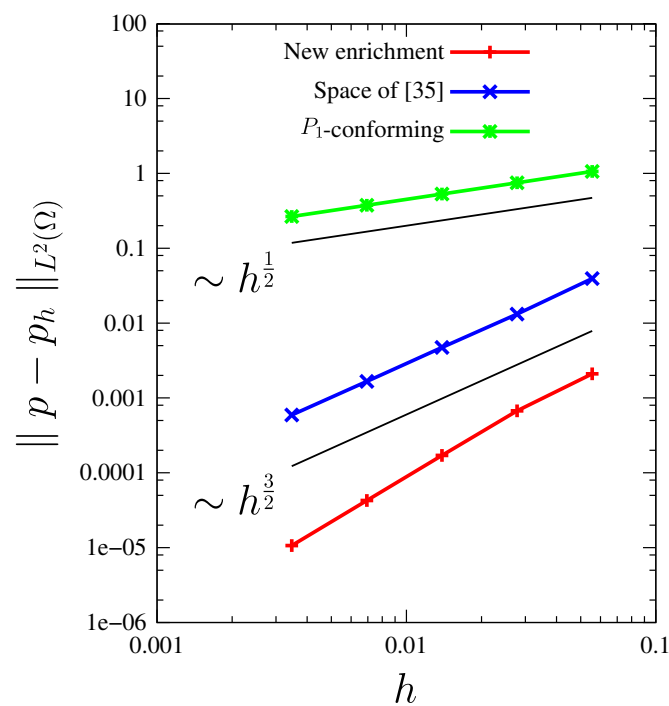

Figure 9. Error norm for the pressure field, showing the convergence rates for the extensional flow problem.

solution belongs to the finite element spaces when either, the new enrichment space or the space of [35] are used.

\section{PROBLEMS WITH SURFACE TENSION}

In the previous academic examples, the interface $\Gamma$ was fixed. Now, we aim to illustrate the use of the new enrichment space in a more complex situation. In this article, a level set formulation is used, in which the interface is the zero set of a continuous scalar function $\phi$, i.e.

$$
\Gamma=\{\mathbf{x} \in \Omega, \phi(\mathbf{x})=0\} .
$$

The level set function is transported according to the following hyperbolic equation

$$
\partial_{t} \phi+\mathbf{u} \cdot \nabla \phi=0 .
$$




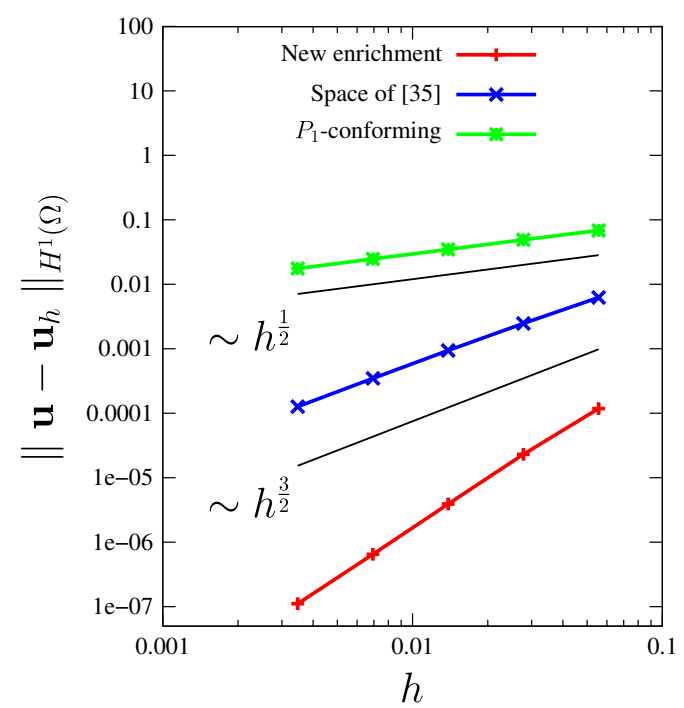

Figure 10. Error norm for the velocity, showing the convergence rates for the extensional flow problem.

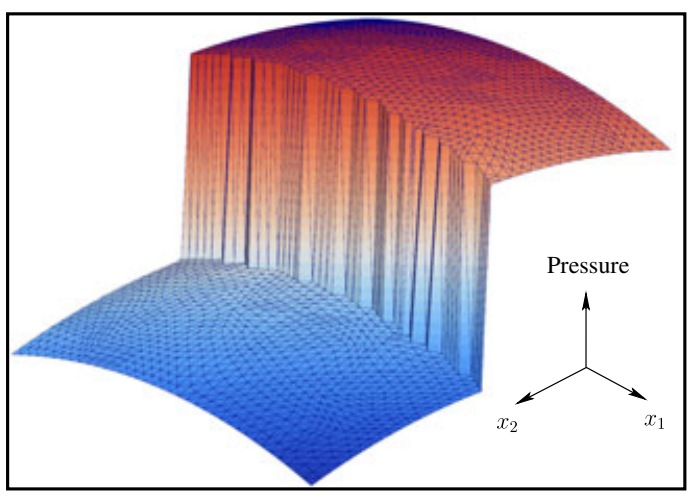

(a) New enrichment space

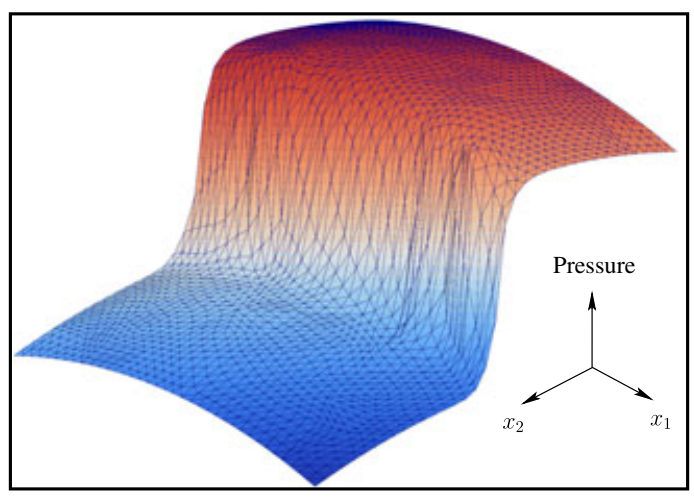

(b) $P_{1}$-conforming space

Figure 11. Pressure fields for the extensional flow problem. (a) New enrichment space. (b) $P_{1}$-conforming space.

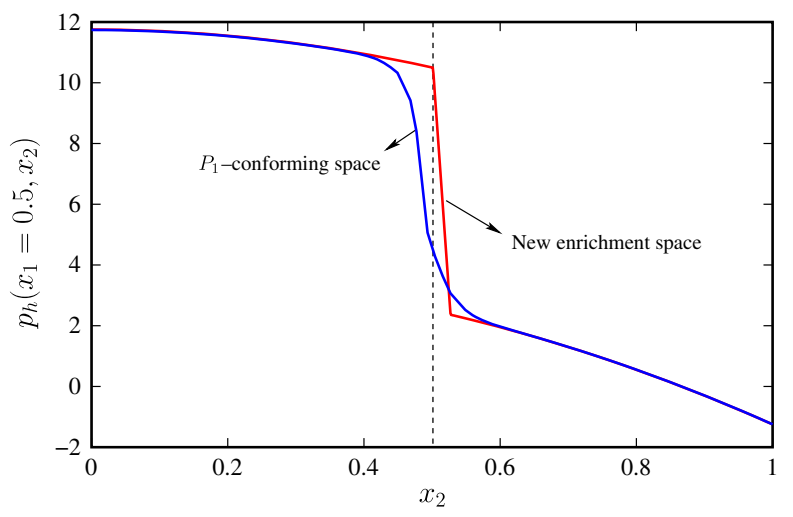

Figure 12. Comparison of the pressure field section at $x_{1}=0.5$ for the extensional flow problem using the new enrichment space and the $P_{1}$-conforming space. 
In the discrete case, the transport equation is solved simultaneously with the velocity and pressure fields by means of an SUPG (streamline-upwind-Petrov-Galerkin) method (see [49]), by adding the following problem to the variational formulation in Equations (19)-(20)

Find $\phi_{h}^{n+1} \in W_{h}$ such that

$$
\sum_{K \in \mathcal{T}_{h}} \int_{\Omega_{K}}\left(\frac{\phi_{h}^{n+1}-\phi_{h}^{n}}{\Delta t}+\mathbf{u}_{h}^{n+1} \cdot \nabla \phi_{h}^{n+1}\right)\left(w_{h}+\tilde{\tau}_{K} \mathbf{u}_{h}^{n+1} \cdot \nabla w_{h}\right) \mathrm{d} \Omega=0
$$

$\forall w_{h} \times W_{h}$. The discrete space $W_{h}$ is made up of continuous linear functions. The stabilization parameter $\tilde{\tau}_{K}$ is given by

$$
\tilde{\tau}_{K}=\frac{\tilde{c} h}{2\left|\mathbf{u}_{h}\right|_{\infty}},
$$

where the factor $\tilde{c}$ is taken equal to 0.1 .

With this formulation, we study the rise of a buoyant bubble. This problem has been solved many times before (see e.g., [50] and references therein). We assume a bubble with density $\rho_{1}=10^{-3}$ and viscosity $\mu_{1}$ in a quiescent liquid with density $\rho_{2}=1$ and viscosity $\mu_{2}$ in the computational domain $\Omega=(0,2.25) \times(0,2.25) \times(0,4)$. At the initial time, the diameter $D$ of the bubble is 1 and is placed at the position $(1.125,1.125,1)$. The gravity $\mathbf{g}$ is taken equal to $-10 \hat{e}_{2}$.

The Morton and Weber numbers are used to characterize the bubble's behavior and are defined as

$$
\mathrm{Mo}=\frac{\|\mathbf{g}\| \mu_{2}^{4}}{\rho_{2} \gamma^{3}}, \quad \mathrm{We}=\frac{\rho_{2}\|\mathbf{g}\| D^{2}}{\gamma}
$$

The different regimes and shapes adopted by the bubbles can be identified in the well-known Grace's diagram [51]. We consider in this paper two different regimes corresponding to the following physical parameters

- Spherical regime: $\mu_{1}=3 \times 10^{-4}, \mu_{2}=0.3, \gamma=10 \Rightarrow \mathrm{Mo}=8.1 \times 10^{-5}$, We $=1$

- Skirted regime: $\mu_{1}=10^{-3}, \mu_{2}=0.1, \gamma=0.1 \Rightarrow \mathrm{Mo}=1, \mathrm{We}=100$

In the first case, labeled spherical regime, the problem is dominated by surface tension effects and the bubble's shape remains approximately spherical during its evolution. In the second case, labeled skirted regime, surface tension effects are less important, and the bubble suffers a larger deformation during the evolution.

The discrete variational formulation with the new enrichment space was included into a general purpose in-house code, which is described elsewhere (see [52]). The level set function is periodically reinitialized by means of a mass-preserving redistancing scheme (see $[53,54])$ to keep its distortion under control. For this problem, the finite element mesh used consists of 1, 511, 016 tetrahedra, and the time step is taken equal to $5 \times 10^{-4}$ for both regimes.

For the spherical regime, plotted in Figure 13 are the interface shapes at times $t=0,0.375$ and 0.75 . The interface is painted with the velocity magnitude. The better mass conservation of the new enrichment space as compared with the classical $P_{1}$-conforming space are evident. In the former case, a $2 \%$ of the bubble's mass is lost at the final time, against a $60 \%$ in the case without enrichment.

For the skirted regime, to better appreciate the benefits of the new enrichment space, we plot in Figure 14 the bubbles at different times. We observe the typical cap shape attained by the bubble. For the case using the new enrichment, the bubble is plotted on the left, in red, whereas for the case using the classical $P_{1}$-conforming space, the bubble is plotted on the right, in blue. The mass loss is $0.14 \%$ in the first case and $15 \%$ in the second one, clearly evidencing the benefits of the new pressure space. For both regimes, similar results to those corresponding to the new enrichment are obtained if the space of [35] is used instead.

Finally, in Figure 15, we plot the position of the bubble's center of mass as a function of time. In this figure, we notice that after an initial transient the center of mass reaches an approximately constant velocity. With these velocities, a Reynolds number can be computed and compared with 

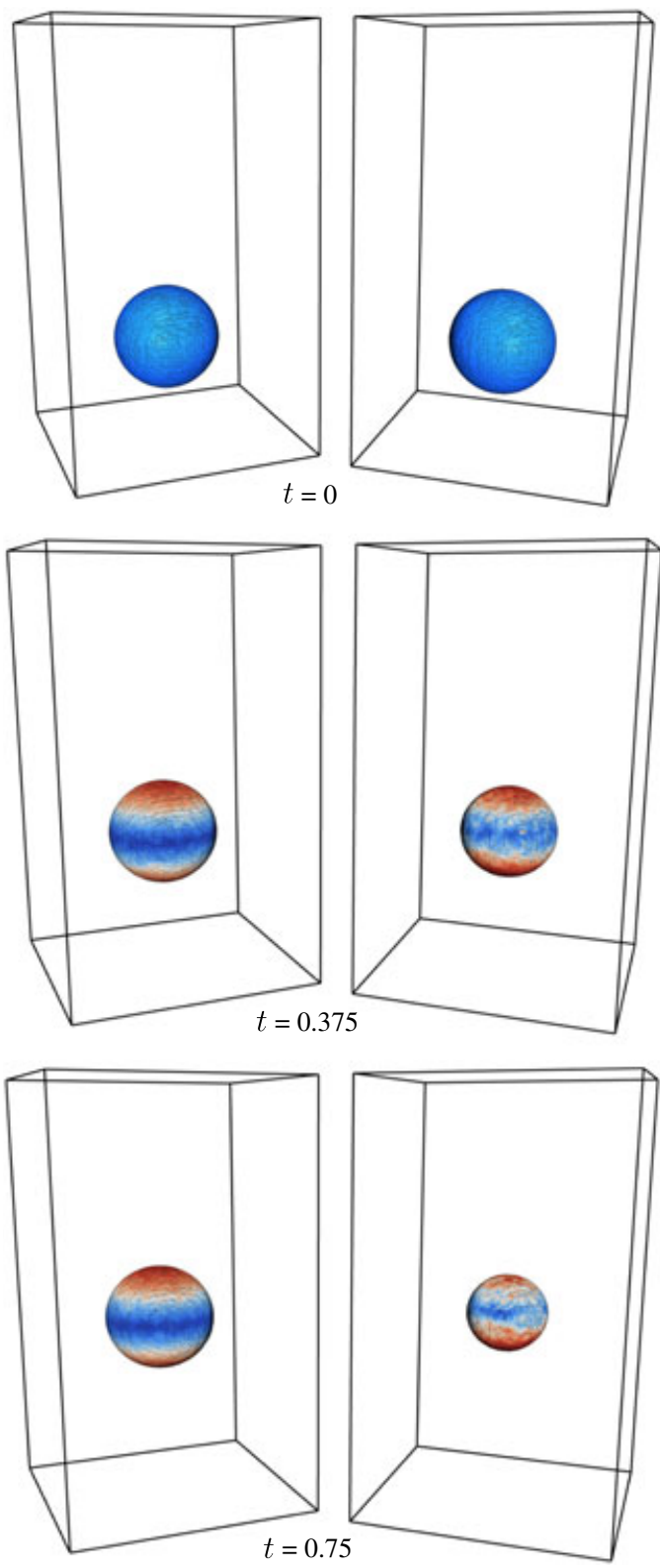

Figure 13. Comparison of the three-dimensional rising bubble at different times for the spherical regime, using the new enrichment space (left) and the classical $P_{1}$-conforming space (right). For times 0.375 and 0.75 , the maximum of the color scale corresponds to 1.3 (red) and the minimum to 0.02 (blue).

the values reported in Grace's diagram [51] as performed by other authors (see e.g., [50]). In Table III, we present the Reynolds number $\mathrm{Re}_{e}$ obtained from Grace's diagram and the computed one $\operatorname{Re}_{c}$ obtained by performing a linear regression of the data shown in Figure 15 starting from time $t=0.4$. Results presented in Table III show a difference of about $20 \%$ between experimental and numerical results.

\section{FURTHER ENRICHMENTS}

We now discuss two further enrichments to improve accuracy of the numerical approximation in problems involving discontinuities and/or kinks. On the one hand, we combine the enrichment 

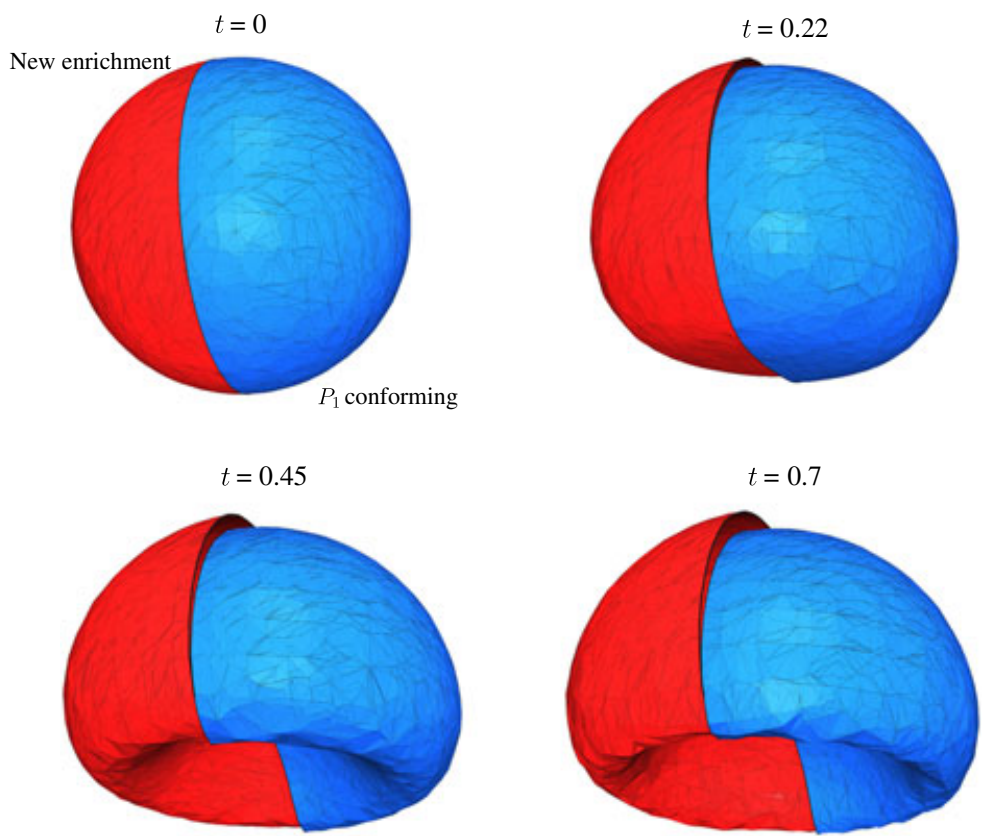

Figure 14. Comparison of the three-dimensional rising bubbles at different times for the skirted regime, using the new enrichment space (left, red) and the classical $P_{1}$-conforming space (right, blue).

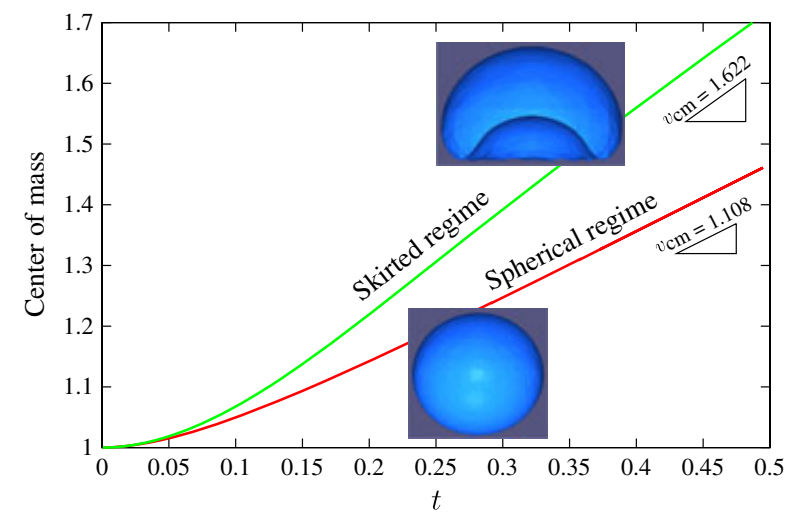

Figure 15. Bubble's center of mass as a function of time for the two regimes considered using the new enrichment space.

Table III. Physical parameters for the two regimes considered for the rise of a buoyant bubble in $3 \mathrm{D}$.

\begin{tabular}{lcrrr}
\hline Regime & Mo & We & $\mathrm{Re}_{e}$ & $\operatorname{Re}_{c}$ \\
\hline Spherical & $8.1 \times 10^{-5}$ & 1 & 5 & 3.93 \\
Skirted & 1 & 100 & 20 & 16.22 \\
\hline
\end{tabular}

function of Codina-Coppola-Owen [44] (for kinks in the pressure field) and the new enrichment functions proposed in this paper leading to a triple enrichment. We illustrate the triple enrichment by means of two numerical examples. On the other hand, we propose without testing it, an enrichment of the velocity field to capture discontinuities in its gradient. Its evaluation is the subject of ongoing work. 


\subsection{Enrichment space of Codina-Coppola-Owen for kinks in the pressure field}

For completeness, we recall here the enrichment proposed in [44], which adds one shape function local to the elements crossed by the interface. This function can be easily defined with the help of a level set function $\psi_{h}$ linearly interpolated on $\Omega_{K}$ and with the usual $P_{1}$ functions as follows

$$
M_{0}(\mathbf{x})=\frac{1}{2}\left(-\left|\psi_{h}(\mathbf{x})\right|+\sum_{J=1}^{n_{p}}\left|\psi_{h}\left(\mathbf{x}_{J}\right)\right| N_{J}(\mathbf{x})\right),
$$

This function is defined as zero outside $\Omega_{K}$ and can thus be condensed before assembly. The enrichment function is illustrated for a typical triangular element in Figure 16, but the extension for the case of tetrahedral elements is straightforward as shown in Table IV where the code to calculate the shape function is included.

It is not difficult to see that the enrichment functions $M_{0}, M_{1}$ and $M_{2}$ are linearly dependent when the interface $\Gamma_{h}$ becomes parallel to one of the element faces. Although this is very unlikely to happen during a general dynamical calculation, the linear independence of the finite element basis may be deteriorated if the interface becomes nearly parallel to one of the faces, resulting in a $3 \times 3$ matrix $A_{M M}$ in Equation (27) approximately singular. This can be overcome by shifting the diagonal entries of $\mathrm{A}_{M M}$ by a small number when this situation is detected along a calculation,

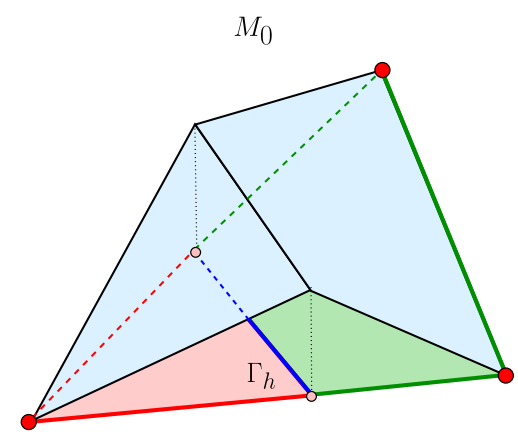

Figure 16. Codina-Coppola-Owen's enrichment function proposed in [44] in a typical triangular element.

Table IV. Computation of the enrichment function $N_{0}^{e}$ for the space of [44]. Nomenclature: $n_{p}$ is the number of nodes per element, $n_{G}$ is the number of integration points per subelement, $N_{J}$ is the usual $J$ th linear basis function, $M_{0}$ is the new enrichment function, $\boldsymbol{X}_{J}^{K}$ are the vertices coordinates of element $\Omega_{K}$ and $\psi_{h}$ is the linearly interpolated level set function on $\Omega_{K}$.

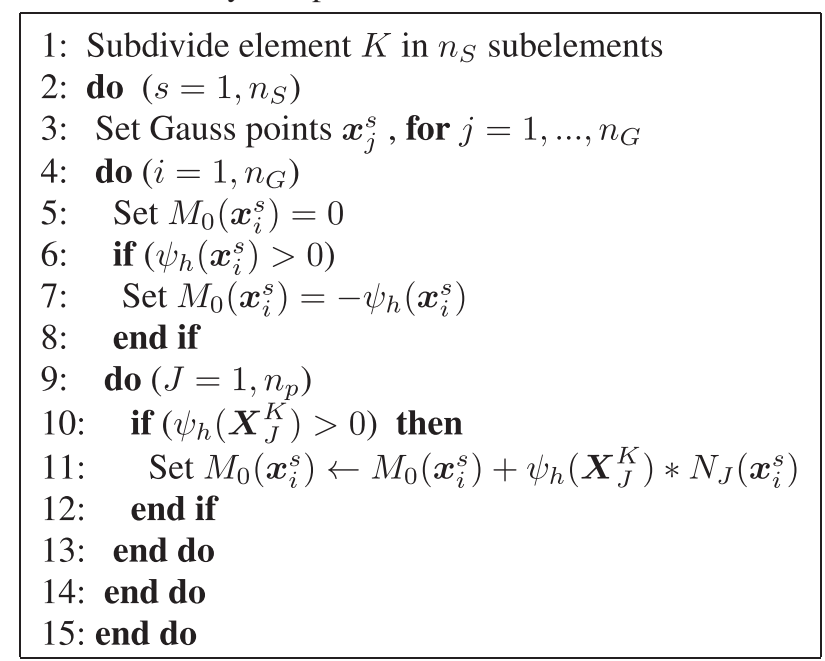


that is,

$$
\mathrm{A}_{M M} \leftarrow \mathrm{A}_{M M}+\varepsilon \mathrm{l},
$$

where $\mathrm{I}$ is the $3 \times 3$ identity matrix and $\varepsilon$ is a small parameter that for the problems presented in this paper we choose as $10^{-4} \sum_{I, J}\left|A_{M M}^{I J}\right|$.

\subsection{Kinks and discontinuities in the pressure field}

\section{Two-fluid hydrostatic problem}

The prototypical example in which there is a discontinuity in the pressure field and its gradient is the case of a two-fluid hydrostatic problem. We consider two fluids in a unit square domain with densities $\rho_{1}$ and $\rho_{2}$ and separated by the fixed interface $x_{2}=a$ (the lighter fluid on the top of the other). At the interface, we introduce a singular force $f_{\Gamma}$ of size 100 as shown in previous examples. The gravity $g$ is taken equal to -10 , the viscosity $\mu$ equal to 1 for both fluids, $\rho_{1}=100, \rho_{2}=1$ and $a=0.5$. Non-slip boundary conditions are used at all walls. The exact solution for this problem consists in a velocity field identically zero and a pressure field given by

$$
p\left(x_{1}, x_{2}\right)= \begin{cases}-\frac{1}{2}\left(\rho_{1}+\rho_{2}\right) g+\rho_{1} g x_{2}+f_{\Gamma} & \text { if } x_{2}<\frac{1}{2} \\ \rho_{2} g\left(x_{2}-1\right) & \text { if } x_{2}>\frac{1}{2}\end{cases}
$$

Note that, there is a discontinuity in the pressure field of size $f_{\Gamma}$ and a discontinuity in its gradient of size $\left(\rho_{1}-\rho_{2}\right)|g|=990$. In Table $\mathrm{V}$, we show the $L^{2}(\Omega)$ and the $H^{1}(\Omega)$ error norms for the pressure and the velocity fields respectively, for two different meshes with characteristic sizes $h=0.0275$ and $h=0.006875$ (the second and fourth meshes of the sequence used in problem 4.2) and using different finite element spaces. We consider, on the one hand, the combination of the enrichment of Codina-Coppola-Owen with the new enrichment space and the combination of the enrichment of Codina-Coppola-Owen with the space of [35]. In the first case, the error is almost zero because the exact solution simply belongs to the finite element space. In the second and third cases, the approximation error is significantly bigger because the exact solution is not in the approximation space.

\section{Extensional flow with discontinuous volume force}

We finally consider the same problem shown in section 4.2 but in this case we introduce a discontinuous volume force as follows

$$
\mathbf{b}= \begin{cases}-10 \mathbf{e}_{2} & \text { if } x_{2}<a \\ 0 & \text { if } x_{2}>a\end{cases}
$$

As a result, the pressure field and its gradient are discontinuous, for which the triple enrichment used before becomes suitable. The pressure field for this problem is given by

$$
p\left(x_{1}, x_{2}\right)=\rho\left(x_{1}-\frac{1}{2}\left(x_{1}^{2}+x_{2}^{2}\right)-|\mathbf{b}| x_{2}\right)+2\left(\mu_{1}-\mu_{2}\right) \mathcal{H}\left(a-x_{2}\right)
$$

in which there is a discontinuity of the pressure gradient of size $\rho|\mathbf{b}|$ and a discontinuity in the pressure of size $2\left(\mu_{1}-\mu_{2}\right)$. In Table VI, we show the $L^{2}(\Omega)$-error norm for the pressure comparing the

Table V. Error norms of pressure and velocity on two different meshes for the two-fluid hydrostatic problem.

\begin{tabular}{lccccc}
\hline & \multicolumn{2}{c}{$h=0.0275$} & & \multicolumn{2}{c}{$h=0.006875$} \\
\cline { 2 - 3 } \cline { 5 - 6 } Pressure space & $\left\|\mathbf{u}-\mathbf{u}_{h}\right\|_{H^{1}(\Omega)}$ & $\left\|p-p_{h}\right\|_{L^{2}(\Omega)}$ & & $\left\|\mathbf{u}-\mathbf{u}_{h}\right\|_{H^{1}(\Omega)}$ & $\left\|p-p_{h}\right\|_{L^{2}(\Omega)}$ \\
\hline New space + [44] & $10^{-13}$ & $10^{-13}$ & & $10^{-13}$ & $10^{-13}$ \\
New space alone & 0.0206 & 0.1569 & & 0.0023 & 0.0181 \\
Space of [35] + [44] & 0.1106 & 0.7574 & & 0.0146 & 0.1018 \\
$P_{1}$ space alone & 8.0647 & 1.3995 & & 3.9313 & 0.7456 \\
\hline
\end{tabular}


Table VI. $L^{2}(\Omega)$-error norm of the pressure as a function of the mesh size. Initial mesh with $h_{0}=0.056$.

\begin{tabular}{lccccc}
\hline Pressure space & $h_{0}$ & $h_{0} / 2$ & $h_{0} / 4$ & $h_{0} / 8$ & $h_{0} / 16$ \\
\hline New space + [44] & $1.73 \times 10^{-3}$ & $5.84 \times 10^{-4}$ & $1.44 \times 10^{-4}$ & $3.56 \times 10^{-5}$ & $8.91 \times 10^{-6}$ \\
New space alone & $5.98 \times 10^{-2}$ & $2.35 \times 10^{-2}$ & $7.95 \times 10^{-3}$ & $2.83 \times 10^{-3}$ & $1.02 \times 10^{-3}$ \\
Space of [35] + [44] & $2.55 \times 10^{-1}$ & $8.97 \times 10^{-2}$ & $3.31 \times 10^{-2}$ & $1.21 \times 10^{-2}$ & $4.21 \times 10^{-3}$ \\
\hline
\end{tabular}

use of the new enrichment and the space of [35], both combined with the enrichment of [44]. Also shown in the table is the case corresponding to the new enrichment alone. The pressure field being a quadratic function, the exact solution does not belong to the finite element space. From the table we observe that the order of convergence for the pressure field is proportional to $h^{2}$ in the first case and proportional to $h^{3 / 2}$ in the second one. Besides this difference, again the new enrichment space exhibits significantly smaller errors.

\subsection{Kinks in the velocity field}

Although it has not been tested yet, using the enrichment functions proposed by Codina-CoppolaOwen, a new enrichment of the velocity field to capture kinks in the velocity field, for problems involving Marangoni effects or two phase flows with $\mu_{1} / \mu_{2} \gg 1$, can be devised. The idea in this case is to enrich each component of the velocity field as follows

$$
u_{i}(\mathbf{x})=\sum_{J \in \mathcal{J}} U_{i J} N_{J}(\mathbf{x})+c_{i} M_{0}(\mathbf{x})
$$

This new degree of freedom is not in $H^{1}(\Omega)$ and thus renders the approximation non-conforming. It could well happen that the errors introduced by this 'variational crime' are small, except perhaps in regions with high curvature for which local mesh refinement may be needed. The evaluation of this enrichment is still the subject of ongoing work.

\section{CONCLUSIONS}

A new enrichment space has been proposed to accommodate discontinuities in the pressure field for problems involving jumps at internal interfaces in multi-fluid flows. The new space consist of adding two degrees of freedom that are local to each element and can thus be condensed prior to assembly.

The new space has been tested and compared with other pressure spaces to accommodate discontinuities at immersed boundaries in several problems involving jumps in the pressure field and its gradient. In the classical Couette flow problem with a singular force the $L^{2}(\Omega)$ and $H^{1}(\Omega)$ error norms for pressure and velocity, respectively, exhibited practically the same results as using the space of [35]. For the extensional flow problem, the errors were much smaller (more than one order of magnitude) and with a better order of convergence for the new enrichment space as compared with the space of [35]. The new enrichment space has also been tested in a more challenging 3D problem involving a rising bubble in different regimes, exhibiting excellent results in terms of mass conservation as compared with the classical $P_{1}$-conforming space.

The possibility of combining the new enrichment with the enrichment proposed in [44] has also been explored in problems involving discontinuities and kinks in the pressure field because of the presence of singular forces and jumps in the density and/or in the volume forces. In this case, care has to be taken to avoid loss of the linear independence of the finite element basis, which can occur when the interface becomes parallel to one of the element faces.

The new proposed enrichment space is an attractive alternative to other existing finite element spaces to accommodate jumps at immersed boundaries. 


\section{ACKNOWLEDGEMENTS}

The authors acknowledge partial support from FAPESP and CNPq (Brazil) and from the ERC Advanced Grant "Real Time - Project AdG_2009325". The authors would also like to thank the International Center for Numerical Methods in Engineering for kindly supporting this work.

\section{REFERENCES}

1. Sommerfeld M, van Wachem B, Oliemans R. Special interest group on dispersed turbulent multi-phase flow, best practice guidelines. ERCOFTAC, 2007.

2. Hughes TJR, Liu WK, Zimmermann TK. Lagrangian-Eulerian finite element formulation for incompressible viscous flows. Computer Methods in Applied Mechanics and Engineering 1981; 29:329-349.

3. Donea J, Huerta A, Ponthot J-P, Rodríguez Ferran A. Arbitrary Lagrangian-Eulerian methods (Stein E, de Borst R, Hughes T, eds), Encyclopedia of Computational Mechanics, Vol. 1. John Wiley \& Sons: New York, 2004.

4. Hirt CW, Amsden AA, Cook JL. An arbitrary Lagrangian-Eulerian computing method for all flow speeds. Journal of Computational Physics 1974; 14:227-253.

5. Cruchaga MA, Calentano DJ, Tezduyar TE. A moving Lagrangian interface technique for flow computations over fixed meshes. Computer Methods in Applied Mechanics and Engineering 2001; 191:525-543.

6. Dettmer W, Saksono PH, Perić D. On a finite element formulation for incompressible Newtonian fluid flows on moving domains in the presence of surface tension. Communications in Numerical Methods in Engineering 2003; 19:659-668.

7. Baiges J, Codina R, Coppola-Owen H. The fixed-mesh ALE approach for the numerical simulation of floating solids. International Journal for Numerical Methods in Fluids 2011; 67(8):1004-1023. DOI: http://dx.doi.org/10.1002/ fld.2403.

8. Idelsohn SR, Oñate E, Del Pin F. The particle finite element method: a powerful tool to solve incompressible flows with free-surfaces and breaking waves. International Journal for Numerical Methods in Engineering 2004; 61:964-989.

9. Idelsohn SR, Mier-Torrecilla M, Oñate E. Multi-fluid flows with the particle finite element method. Computer Methods in Applied Mechanics and Engineering 2009; 198:2750-2767.

10. Mier-Torrecilla M, Idelsohn SR, Oñate E. Advances in the simulation of multi-fluid flows with the particle finite element method. International Journal for Numerical Methods in Fluids 2010. DOI: http://dx.doi.org/10.1002/fld.2429. Published online.

11. Idelsohn SR, Mier-Torrecilla M, Nigro N, Oñate E. On the analysis of heterogeneous fluids with jumps in the viscosity using a discontinuous pressure field. Computational Mechanics 2010; 46(1):115-124.

12. Unverdi S, Tryggvason G. A front-tracking method for viscous, incompressible, multi-fluid flows. Journal of Computational Physics 1992; 100:25-37.

13. Gueyffier D, Lie J, Nadim A, Scardovelli R, Zaleski S. Volume-of-fluid interface tracking with smoothed surface stress methods for three-dimensional flows. Journal of Computational Physics 1999; 152:423-456.

14. Popinet S, Zaleski S. A front-tracking algorithm for accurate representation of surface tension. International Journal for Numerical Methods in Fluids 1999; 30:775-793.

15. Hirt CW, Nichols HD. Volume of fluid (VOF) methods for the dynamics of free boundaries. Applied Numerical Mathematics 1981; 39:201-225.

16. Kothe DB, Rider WJ, Mosso SJ, Brock JS, Hochstein JI. Volume tracking of interfaces having surface tension in two and three dimensions. Technical Report AIAA 96-0859, AIAA, 1996. Presented at the 34rd Aerospace Sciences Meeting and Exhibit.

17. Cummins SJ, Francois MM, Kothe DB. Estimating curvature from volume fraction. Computers and Structures 2005; 83:425-434.

18. Adalsteinsson D, Sethian J. A fast level set method for propagating interfaces. Journal of Computational Physics 1995; 118:269-277.

19. Sethian JA. Evolution, implementation and application of level set and fast marching methods for advancing fronts. Journal of Computational Physics 2001; 169:503-555.

20. Osher S, Fedkiw R. Level set methods: an overview and some recent results. Journal of Computational Physics 2001; 169:463-502.

21. Guermond JL, Quartapelle L. A projection FEM for variable density incompressible flows. Journal of Computational Physics 2000; 165:167-188.

22. Shu C, Osher S. Efficient implementation of essentially non-oscillatory shock-capturing schemes. Journal of Computational Physics 1988; 77:439-471.

23. Jiang G-S, Peng D. Weighted ENO schemes for Hamilton-Jacobi equations. SIAM Journal on Scientific Computing 2000; 21:2126-2144.

24. Sweby PK. High resolution schemes using flux limiters for hyperbolic conservation laws. SIAM Journal on Numerical Analysis 1984; 21:995-1011.

25. Enright D, Fedkiw R, Ferziger J, Mitchell I. A hybrid particle level set method for improved interface capturing. Computers and Structures 2002; 83:479-490. 
26. Marchandise E, Remacle J-F, Chevaugeon N. A quadrature-free discontinuous Galerkin method for the level set equation. Journal of Computational Physics 2006; 212:338-357.

27. Di Pietro D, Lo Forte S, Parolini N. Mass preserving finite element implementations of the level set method. Applied Numerical Mathematics 2006; 56:1179-1195.

28. Enright D, Losasso F, Fedkiw R. A fast and accurate semi-Lagrangian particle level set method. Computers and Structures 2005; 83:479-490.

29. Strain J. Semi Lagrangian methods for level set equations. Journal of Computational Physics 1999; 151:498-533.

30. Strain J. Tree methods for moving interfaces. Journal of Computational Physics 1999; 151:616-648.

31. Brackbill JU, Kothe DB, Zemach C. A continuum method for modeling surface tension. Journal of Computational Physics 1992; 100:335-354.

32. Löhner R, Yang C, Oñate E. On the simulation of flows with violent free surface motion. Computer Methods in Applied Mechanics and Engineering 2006; 195:5597-5620.

33. Carrica P, Wilson R, Stern F. An unsteady single-phase level set method for viscous free surface flows. International Journal of Numerical Methods in Fluids 2007; 53:229-256.

34. Ganesan S, Matthies G, Tobiska L. On spurious velocities in incompressible flow problems with interfaces. Computer Methods in Applied Mechanics and Engineering 2007; 196:1193-1202.

35. Ausas RF, Sousa FS, Buscaglia GC. An improved finite element space for discontinuous pressures. Computer Methods in Applied Mechanics and Engineering 2010; 199:1019-1031.

36. Buscaglia G, Agouzal A. Interpolation estimate for a finite element space with embedded discontinuities. IMA Journal of Numerical Analysis, accepted 2011. DOI: 10.1093/imanum/drq045.

37. Minev PD, Chen T, Nandakumar K. A finite element technique for multifluid incompressible flow using Eulerian grids. Journal of Computational Physics 2003; 187:255-273.

38. Chessa J, Belytschko T. An extended finite element method for two-phase fluids. Journal of Applied Mechanics 2003; 70:10-17.

39. Belytschko T, Moës N, Usui S, Parimi C. Arbitrary discontinuities in finite elements. International Journal for Numerical Methods in Engineering 2001; 50:993-1013.

40. Gross S, Reusken A. Finite element discretization error analysis of a surface tension force in two-phase incompressible flows. SIAM Journal on Numerical Analysis 2007; 45:1679-1700.

41. Gross S, Reusken A. An extended pressure finite element space for two-phase incompressible flows with surface tension. Journal of Computational Physics 2007; 224:40-58.

42. Reusken A. Analysis of an extended pressure finite element space for two-phase incompressible flows. Computing and Visualization in Science 2008; 11:293-305.

43. Fries T-P, Belytschko T. The intrinsic XFEM: a method for arbitrary discontinuities without additional unknowns. International Journal for Numerical Methods in Engineering 2006; 68:1358-1385.

44. Coppola-Owen AH, Codina R. Improving eulerian two-phase flow finite element approximation with discontinuous gradient pressure shape functions. International Journal for Numerical Methods in Fluids 2005; 49:1287-1304.

45. Buscaglia GC, Ausas RF. Variational formulations for surface tension, capillarity and wetting. Computer Methods in Applied Mechanics and Engineering 2011; 200(45-46):3011-3025.

46. Bänsch E. Finite element discretization of the Navier-Stokes equation with a free capillary surface. Numerische Mathematik 2001; 88:203-235.

47. Codina R. A stabilized finite element method for generalized stationary incompressible flows. Computer Methods in Applied Mechanics and Engineering 2001; 190:2681-2706.

48. Chang Y, Hou T, Merriman B, Osher S. A level set formulation of eulerian capturing methods for incompressible fluid flows. Journal of Computational Physics 1996; 124:449-464.

49. Hughes T. Recent progress in the development and understanding of SUPG methods with special reference to the compressible Euler and Navier-Stokes equations. International Journal for Numerical Methods in Fluids 1987; 7:1261-1275.

50. Marchandise E, Geuzaine P, Chevaugeon N, Remacle JF. A stabilized finite element method using a discontinuous level set approach for the computation of bubble dynamics. Journal of Computational Physics 2007; 225:949-974.

51. Clift R, Grace J, Weber M. Bubbles, Drops and Particles. Dover publications: Mineola, New York, 2005.

52. Ausas R. Numerical simulation in two-phase immiscible flows with applications in hydrodynamic lubrication. $P h D$ Thesis, Engineering, Instituto Balseiro, April, 2010.

53. Mut F, Buscaglia G, Dari E. New mass-conserving algorithm for level set redistancing on unstructured meshes. Journal of Applied Mechanics 2006; 73:1011-1016.

54. Ausas RF, Buscaglia GC, Dari EA. A geometric mass-preserving redistancing scheme for the level set function. International Journal for Numerical Methods in Fluids 2011; 65:989-1010. 